\title{
NEAR-ULTRAVIOLET ABSORPTION, CHROMOSPHERIC ACTIVITY, AND STAR-PLANET INTERACTIONS IN THE WASP-12 SYSTEM*
}

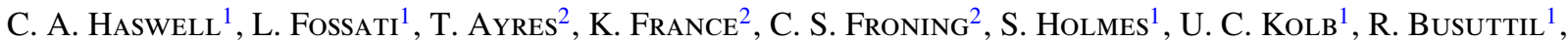 \\ R. A. Street ${ }^{3}$, L. Hebb ${ }^{4}$, A. Collier Cameron ${ }^{5}$, B. Enoch ${ }^{5}$, V. Burwitz ${ }^{6}$, J. Rodriguez ${ }^{7}$, R. G. West ${ }^{8}$, \\ D. Pollacco ${ }^{9}$, P. J. Wheatley ${ }^{9}$, and A. Carter ${ }^{1}$ \\ ${ }^{1}$ Department of Physical Sciences, The Open University, Walton Hall, Milton Keynes MK7 6AA, UK; C.A.Haswell@open.ac.uk, 1.fossati@open.ac.uk \\ ${ }^{2}$ Center for Astrophysics and Space Astronomy, University of Colorado, 593 UCB, Boulder, CO 80309-0593, USA; cynthia.froning @ colorado.edu \\ ${ }^{3}$ Las Cumbres Observatory Global Telescope Network, Inc., 6740 Cortona Drive, Suite 102, Goleta, CA 93117, USA \\ ${ }^{4}$ Department of Physics and Astronomy, Vanderbilt University, 6301 Stevenson Center Nashville, TN 37235, USA; leslie.hebb@vanderbilt.edu \\ ${ }^{5}$ School of Physics and Astronomy, University of St Andrews, North Haugh, St Andrews, Fife KY16 9SS, UK \\ ${ }^{6}$ Max Planck Institut für Extraterrestrische Physik, Giessenbachstrasse, D-85748 Garching, Germany \\ ${ }^{7}$ Observatori Astronómic de Mallorca, Camí de l'Observatori, E-07144 Costitx, Mallorca, Spain \\ ${ }^{8}$ Department of Physics \& Astronomy, University of Leicester, Leicester LE1 7RH, UK \\ ${ }^{9}$ Department of Physics, University of Warwick, Coventry CV4 7AL, UK \\ Received 2012 July 27; accepted 2012 September 27; published 2012 November 6
}

\begin{abstract}
Extended gas clouds have been previously detected surrounding the brightest known close-in transiting hot Jupiter exoplanets, HD 209458 b and HD 189733 b; we observed the distant but more extreme close-in hot Jupiter system, WASP-12, with Hubble Space Telescope (HST). Near-UV (NUV) transits up to three times deeper than the optical transit of WASP-12 b reveal extensive diffuse gas, extending well beyond the Roche lobe. The distribution of absorbing gas varies between visits. The deepest NUV transits are at wavelength ranges with strong stellar photospheric absorption, implying that the absorbing gas may have temperature and composition similar to those of the stellar photosphere. Our spectra reveal significantly enhanced absorption (greater than $3 \sigma$ below the median) at $\sim 200$ individual wavelengths on each of two HST visits; 65 of these wavelengths are consistent between the two visits, using a strict criterion for velocity matching that excludes matches with velocity shifts exceeding $\sim 20 \mathrm{~km} \mathrm{~s}^{-1}$. Excess transit depths are robustly detected throughout the inner wings of the $\mathrm{Mg}$ II resonance lines independently on both $H S T$ visits. We detected absorption in Fe II $\lambda 2586$, the heaviest species yet detected in an exoplanet transit. The Mg II line cores have zero flux, emission cores exhibited by every other observed star of similar age and spectral type are conspicuously absent. WASP-12 probably produces normal $\mathrm{Mg}$ II profiles, but the inner portions of these strong resonance lines are likely affected by extrinsic absorption. The required $\mathrm{Mg}^{+}$column is an order of magnitude greater than expected from the interstellar medium, though we cannot completely dismiss that possibility. A more plausible source of absorption is gas lost by WASP-12 b. We show that planetary mass loss can produce the required column. Our Visit 2 NUV light curves show evidence for a stellar flare. We show that some of the possible transit detections in resonance lines of rare elements may be due instead to non-resonant transitions in common species. We present optical observations and update the transit ephemeris.
\end{abstract}

Key words: planetary systems - planets and satellites: atmospheres - planets and satellites: composition - planets and satellites: individual (WASP-12 b, HD189733 b) - planets and satellites: physical evolution - planet-star interactions - stars: activity - stars: chromospheres - stars: individual (WASP-12, HD189733)

Online-only material: color figures

\section{INTRODUCTION}

WASP-12 b is one of the most extreme of the hot Jupiter exoplanets: it orbits only one stellar diameter away from the photosphere of its late $\mathrm{F}$ type host star, and is thus one of the most irradiated planets known (Hebb et al. 2009). The brightest hot Jupiters, HD $189733 \mathrm{~b}$ and HD $209458 \mathrm{~b}$, are known to be surrounded by extended clouds of absorbing gas which have been detected through observations of their transits in the farUV (FUV; Vidal-Madjar et al. 2003, 2004; Ben-Jaffel 2007; Vidal-Madjar et al. 2008; Lecavelier Des Etangs et al. 2010; Linsky et al. 2010; Lecavelier Des Etangs et al. 2012). These FUV transits occult the patchy and time-variable chromospheric emission from the star (Haswell 2010): the FUV continuum

\footnotetext{
* Based on observations made with the NASA/ESA Hubble Space Telescope, obtained from MAST at the Space Telescope Science Institute, which is operated by the Association of Universities for Research in Astronomy, Inc., under NASA contract NAS 5-26555. These observations are associated with programs 11651 and 11673.
}

from even the nearest transiting-planet host stars is faint (see, e.g., Linsky et al. 2010). The FUV transit light curve therefore depends on the locus the planet follows across this constantly changing irregular chromospheric emission distribution and also depends on the velocity range of the emission line adopted for analysis (Ben-Jaffel 2007; Vidal-Madjar et al. 2008).

Several mechanisms have been proposed to explain the extended absorbing gas. A hydrodynamic "blow-off" of the planet's outer atmosphere caused by the intense irradiation was the first and most obvious (Vidal-Madjar et al. 2004). This model has been extensively discussed and refined, most recently by Guo (2011) and Ehrenreich \& Desert (2011). Adams (2011) considers how these outflows might be influenced by the planetary magnetic field, while Lecavelier Des Etangs et al. (2004), Lecavelier Des Etangs (2007), and Erkaev et al. (2007) consider the effect of the Roche equipotentials on the outflow.

Li et al. (2010) suggested that for eccentric hot Jupiters, tidal disruption might drive mass loss orders of magnitude greater than that from irradiation-driven hydrodynamic outflow. This 
was motivated by the implications in the discovery paper that WASP-12 b had a significantly non-zero eccentricity (Hebb et al. 2009). The most recent measurements of the orbital eccentricity of WASP-12 b are, however, consistent with zero eccentricity (Lopez-Morales et al. 2010; Husnoo et al. 2011), so tidal disruption is not expected to play a significant role for WASP-12 b. It could nonetheless play an important role for hot Jupiters found to have non-zero eccentricity, for example due to perturbations by other planets in the system.

A third explanation proposes that the planet is surrounded by a cloud of energetic neutral atoms caused by interactions with the host star's stellar wind (Holmström et al. 2008; Ekenbäck et al. 2010). Tremblin \& Chiang (2012) simulate these colliding winds reproducing the observed $\operatorname{Ly} \alpha$ transit profiles. Raiput \& Safvan (2011) report the formation of energetic neutral atoms from ion-impact dissociation of water in the laboratory and suggest that this process may contribute to the absorbing gas in hot Jupiter exospheres.

Lai et al. (2010) considered mass loss via Roche lobe overflow in WASP-12, while others (e.g., Vidotto et al. 2010) suggested that the absorbing gas may simply be entrained material from the stellar corona, in which case it provides an opportunity to measure the planetary magnetic field. Llama et al. (2011) model the radiative transfer through compressed material behind the magnetospheric bow shock produced in this scenario.

Since WASP-12 b is such an extreme hot Jupiter, it was an obvious target for observations to examine the properties of the extended absorbing gas around these systems. The UV spectral region contains many resonance lines of common elements, including Ly $\alpha$, which provided the first detection of this phenomenon (Vidal-Madjar et al. 2003). These resonance lines provide a sensitive probe for the presence of absorbing gas. WASP-12's distance is $300 \mathrm{pc}$ or more (Fossati et al. 2010a), and this means that the predicted FUV emission would be extremely hard to detect. Thus, we chose to observe in the near-UV (NUV) using the COS spectrograph on Hubble Space Telescope (HST) to cover the strong $\mathrm{Mg}$ II resonance lines and a host of other spectral lines from a rich variety of chemical elements. Our choice of the NUV spectral region has the additional benefit that our transit light curves are measured using the well-understood intensity distribution from the stellar photosphere, which is expected to remain constant with time. In the NUV, we are also able to detect absorption from gas with any velocity: in the FUV, gas ceases to produce an observable effect once its velocity exceeds the velocity width of the stellar chromospheric line emission which is being absorbed.

Our observations comprised two visits, and we published a short paper on the results from the first visit in Fossati et al. (2010b, hereafter Paper I). In Paper I, we presented light curves revealing an exosphere that appears to overfill its Roche lobe and wavelength resolved data, identifying enhanced transit depths at the wavelengths of a number of resonance lines.

In this paper, we report on our full complement of $H S T / C O S$ NUV observations of WASP-12 and on contemporaneous ground-based optical photometry. In Section 2, we describe our observations and data reduction. Section 3 gives our observational results and develops their interpretation. Section 4 critically examines the interpretation suggested by our observational results and presents calculations demonstrating their plausibility. While the conclusions of Paper I are not significantly changed by our re-examination with the benefit of more extensive data, the new data demand a more complex interpretation than our first analysis in Paper I suggested.
Table 1

The Temporal and Wavelength Coverage of Our Two HST/COS Visits

\begin{tabular}{ccc}
\hline \hline Visit & $\begin{array}{c}\text { Wavelength } \\
\text { Range }(\AA)\end{array}$ & $\begin{array}{c}\text { Temporal Coverage } \\
\text { (HJD-2,455,000) }\end{array}$ \\
\hline \multirow{3}{*}{1} & NUVA: 2539-2580 & $099.274652-099.296111$ (orb1) \\
& NUVB: 2655-2696 & $099.330823-099.369516$ (orb2) \\
& NUVC: 2770-2811 & $099.397468-099.432689$ (orb3) \\
& & $099.464090-099.499311$ (orb4) \\
& & $099.530735-099.565956$ (orb5) \\
\hline \multirow{3}{*}{2} & NUVA: 2551-2594 & $283.695216-283.722230$ (orb1) \\
& NUVB: 2669-2711 & $283.753387-283.788606$ (orb2) \\
& NUVC: 2789-2829 & $283.819948-283.855168$ (orb3) \\
& & $283.886510-283.921729$ (orb4) \\
& & $283.953072-283.988291$ (orb5)
\end{tabular}

\section{OBSERVATIONS AND DATA REDUCTION}

We observed WASP-12 with HST/COS, and from the ground with PIRATE (Holmes et al. 2011), Faulkes Telescope North, and the James Gregory Telescope (JGT). These observations are described in Sections 2.1, 2.2, 2.3, and 2.4 respectively.

\subsection{HST/COS Observations}

COS is a slitless spectrograph which we used to obtain $R \sim$ 20,000 NUV spectroscopy. COS maximizes the spectroscopic sensitivity of HST in the UV; a full instrument description and on-orbit performance characteristics can be found in Osterman et al. (2011) and Green et al. (2012).

Our HST observations are summarized in Table 1. Visits 1 and 2 each comprised five consecutive $H S T$ orbits and were executed on 2009 September 24/25 and 2010 March 28, respectively. A first analysis and brief discussion of Visit 1 was given in Paper I. For Visit 1, we used the NUV G285M grating at the $2676 \AA$ setting, which provides non-contiguous spectra over three wavelength ranges; see Table 1 . For Visit 2, we used the $2695 \AA$ setting of the same grating, which gives a slight shift to the red as detailed in Table 1. Our set-up for the two visits was otherwise identical, with spectral resolution of $R \sim 20,000$, without the use of offset FP-POS positions. We obtained simultaneous lamp spectra during science exposures using "FLASH = YES."

The settings for Visit 1 optimized coverage of the region around the core of the strong Mg II UV resonance lines; for Visit 2 we chose to cover the Fe II resonance line at $2586 \AA$ while maximizing the overlap in wavelength between the two sets of observations. Obtaining a homogeneous data set may have had advantages, as we are photon starved, and the behavior of the WASP-12 system appears different on the two visits. The wavelength shift means that only a subset of our spectral coverage can be combined to make light curves which can be straightforwardly interpreted. In Sections 3.6 and 4.1, however, we use the Fe II resonance line to disentangle a complicated story, and it is decisive in one of the major interpretative questions of this paper.

In each visit, the exposure time was $2334 \mathrm{~s}$ in the first HST orbit and about $3000 \mathrm{~s}$ per subsequent HST orbit. We designed the timings of the two visits so that they interleave to give full phase coverage of the transit with out-of-transit (OOT) coverage before ingress and after egress. Our observations were obtained in TIME-TAG observing mode, in which individual photon events are recorded at a resolution of $32 \mathrm{~ms}$, but our effective time resolution is much poorer due to the faintness of the target. 


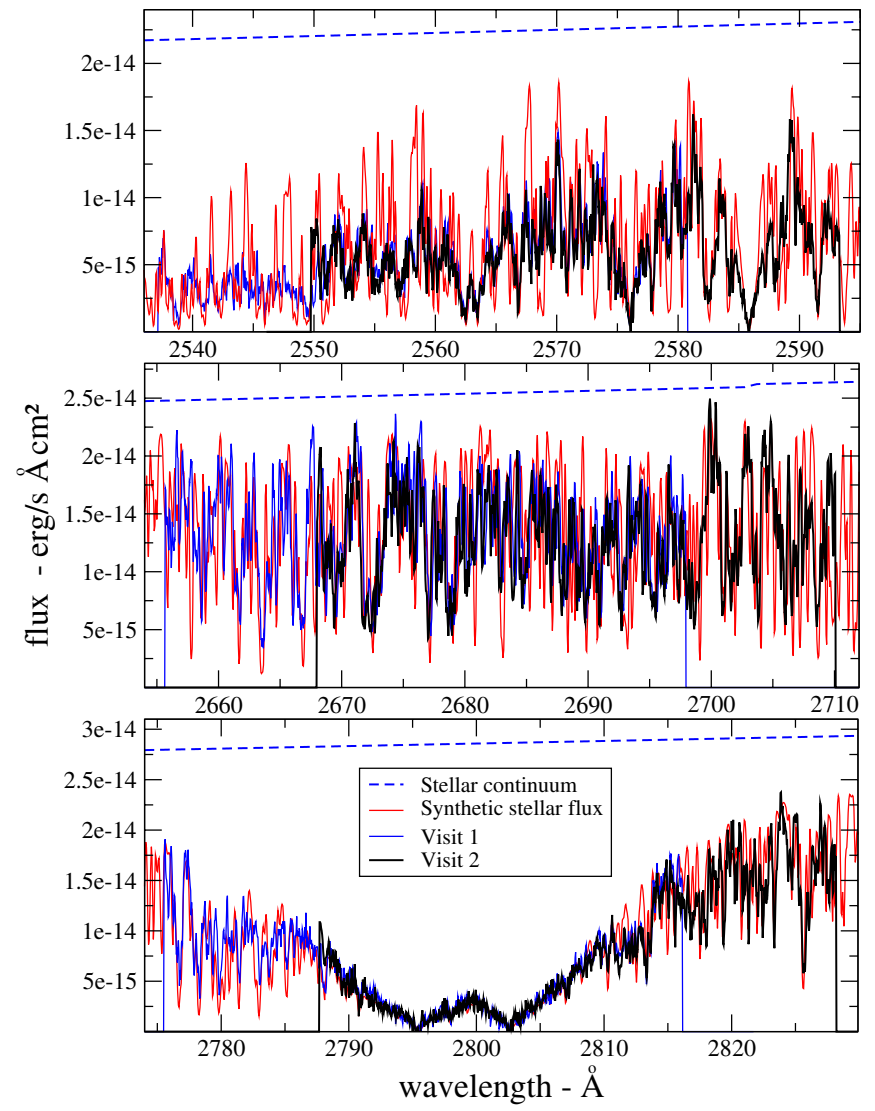

Figure 1. HST/COS NUV spectra of WASP-12 (Visit 1: blue line; Visit 2: black line) and a scaled model WASP-12 stellar spectrum (red line). The stellar continuum (dashed blue line) lies well above the observed flux due to overlapping stellar absorption features throughout the spectrum. The mean flux is slightly lower in Visit 2; see the text. The three panels show the NUVA, NUVB, and NUVC spectral regions.

The count rates summed over wavelength are $\sim 10$ count $\mathrm{s}^{-1}$, $\sim 28$ count $\mathrm{s}^{-1}$, and $\sim 13$ count $\mathrm{s}^{-1}$, respectively, for the NUVA, NUVB, and NUVC ranges (see Table 1 for definitions). The data were reduced using the calibration files provided by STScI; these were updated following Paper I, so we re-reduced all the HST data using the updated calibration files for the present paper. We downloaded data from MAST ${ }^{10}$ adopting CALCOS V.2.11 $\mathrm{b}^{11}$ for calibration. The COS data comprise exposures of the science target with corresponding lamp spectra displaced in the spatial direction. The effects of the new reduction on the data presented in Paper I are shown at the end of this section.

The reduced Visit 2 data as downloaded from the MAST archive suffered from a problem in the extraction of the science spectra and in the determination of the background level. We characterized the location of the science spectra by fitting Gaussians to the profile in the spatial direction. The center of the extraction box for the NUVA region, as defined in the extraction reference file, is about 5 pixels from the central peak of the science spectrum. Similarly, the center of one of the two extraction boxes used by CALCOS to compute the background level is misplaced so that a portion of the science spectrum falls into the background extraction box. To solve this problem, we compared the position of the central pixel of each of the

\footnotetext{
10 http://archive.stsci.edu/

11 See the COS Data Handbook for more information on CALCOS. The version of CALCOS we used did not correct for declining sensitivity, while more recent versions now do.
}

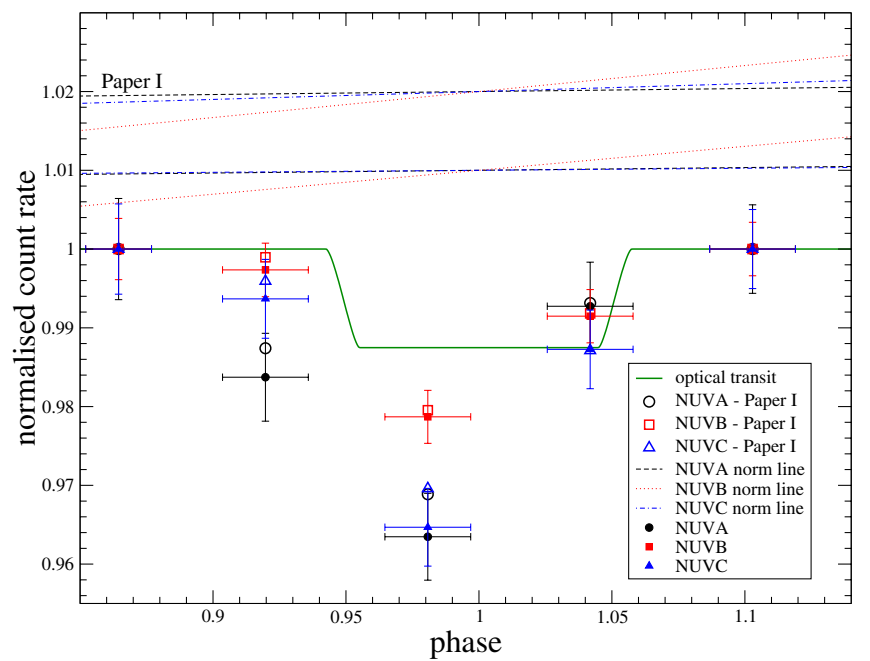

Figure 2. Visit 1 results from Paper I (open symbols) compared to the same data re-reduced with the updated calibration files (closed symbols). The normalization lines indicate the tilt which has been removed from the data in setting the first and last point to unity (i.e., the first two points in the NUVB light curve were moved slightly upward). The upper set of normalization lines correspond to the Paper I reduction. The green line shows the model transit of the optically opaque planet across a uniform brightness stellar disk.

NUVA, NUVB, and NUVC spectra as given in the reference file for different settings of the same grating. The prescribed central pixel differs for different settings, but NUVA, NUVB, and NUVC are always at the same relative positions, except for the $2695 \AA$ setting. For this reason, we simply corrected the extraction box for the NUVA and background region by adopting the same shift as for the NUVB and NUVC regions, we then re-ran CALCOS and the extraction succeeded.

Figure 1 shows the flux-calibrated grand sum spectra obtained in Visit 1 and Visit 2 in comparison with synthetic fluxes, as discussed in Section 3.2. The mean fluxes are $9 \% \pm 1 \%$, $7 \% \pm 1 \%$, and $3.5 \% \pm 1.0 \%$ lower in Visit 2 for NUVA, NUVB, and NUVC, respectively. This is largely attributable to the declining throughput (Osten et al. 2010), but also due to the varying mean obscuration of the WASP-12 stellar flux by the planet and diffuse gas. We will discuss a likely third contributor to varying fluxes in Section 3.6.

In our time-series analysis, we used the count rates obtained after background subtraction, rather than the flux-calibrated spectra. The high quality flat field and the relatively low background of the NUV channel mean that the uncertainties are dominated by Poisson statistics. The resulting signal-tonoise ratio $(\mathrm{S} / \mathrm{N})$ in our extracted NUVB spectra is $\sim 8$ pixel $^{-1}$ for each $3000 \mathrm{~s}$ exposure. This is consistent with the expected photon counting noise and propagates to a fractional uncertainty of $\sim 0.007$ when a $3000 \mathrm{~s}$ exposure is averaged.

Figure 2 shows our Visit 1 light curves. These were produced by summing across wavelength for each of NUVA, NUVB, and NUVC to produce a photometric point for each HST orbit. Figure 2 compares the results from Paper I with those from our revised calibration. The first and last data points for both reductions are set to unity with the normalization procedure applied in Paper I; all the remaining points are within $1 \sigma$ of their previous positions. For all three wavelength regions, the normalization lines (see Paper I) are slightly improved (flatter) with the new calibration files. The normalization applied does not significantly affect the shape of the observed NUV transit, as no point moves by as much as $1 \sigma$. 
Table 2

WASP-12 Observations with PIRATE

\begin{tabular}{lcccc}
\hline \hline $\begin{array}{l}\text { Date } \\
\text { (BON) }\end{array}$ & $\begin{array}{l}\text { HJD Start } \\
-2455000\end{array}$ & $\begin{array}{c}\text { HJD End } \\
-2455000\end{array}$ & $\begin{array}{c}\text { Exp. } \\
\text { Time }(\mathrm{s})\end{array}$ & Comments \\
\hline 2009 Oct 15 & 121.53078 & 121.68142 & $120 \mathrm{~s}$ & OOT only \\
2009 Oct 18 & 123.49117 & 123.67301 & $120 \mathrm{~s}$ & OOT only \\
2009 Nov 11 & 147.47507 & 147.62549 & $120 \mathrm{~s}$ & $\begin{array}{c}\text { Egress and OOT } \\
\text { High humidity (>90\%) }\end{array}$ \\
& & & & OOT and ingress \\
2009 Nov 12 & 148.47187 & 148.51421 & $60 \mathrm{~s}$ & Egress and OOT \\
2009 Nov 22 & 158.37221 & 158.69467 & $60 \mathrm{~s}$ & Mid-transit, egress, and OOT \\
2009 Nov 23 & 159.44126 & 159.59863 & $60 \mathrm{~s}$ & Used PIRATE Mk. 2 \\
2011 Jan 13 & 575.26270 & 575.36230 & $45 \mathrm{~s}$ & Holmes et al. (2011) \\
\hline
\end{tabular}

The NUVA point immediately preceding optical ingress and the NUVA and NUVC points at mid-transit have been moved down almost $1 \sigma$ relative to the reduction presented in Paper I. The refined reduction therefore strengthens the case (made in Paper I) for an early ingress and an extended asymmetric cloud of absorbing gas: the NUVA and NUVC transit depths are now $\sim 4 \sigma$ below the depth of the optical transit. When we consider the data from both visits together, however, these conclusions become more complicated, as discussed in Section 3.3.

\subsection{PIRATE Optical Photometry}

In conjunction with our first $H S T$ visit, we undertook a program of optical photometry with The Open University's remotely operable optical telescope, PIRATE (Holmes et al. 2011). The goal of these observations was to verify or, if necessary update, the WASP-12 b orbital ephemeris. The PIRATE observations are logged in Table 2 . We used the $R$ filter on all seven nights, and either $120 \mathrm{~s}, 60 \mathrm{~s}$, or $45 \mathrm{~s}$ exposures. The dead time between exposures is $15 \mathrm{~s}$, resulting in a cadence of $135 \mathrm{~s}$, $75 \mathrm{~s}$, or $60 \mathrm{~s}$. We captured one ingress, four egresses, and on two nights OOT coverage only. The control computer's real-time clock was synchronized every hour with a USNO time server, typically requiring $11-13 \mathrm{~s}$ correction each time. Light curve precision was 2.5-2.8 mmag, which we achieved through an iterative comparison-ensemble procedure (Holmes et al. 2011). The light curve of one egress is published in Holmes et al. (2011, their Figure 7), and we present our phase-folded optical light curve in Section 3.1 below.

\subsection{Faulkes Telescope North Optical Photometry}

The Spectral camera on the $2 \mathrm{~m}$ Faulkes Telescope North, Hawai'i, was used to observe WASP-12 on 2010 December 17 UT through a Bessell-B filter. The Spectral camera is a $4096 \times 4096$ pixel Fairchild CCD486 BI which was used in bin $2 \times 2$ mode giving a 0.304 arcsec pixel $^{-1}$ scale over the $10 \times 10$ arcmin field of view (FOV). We used a $22 \mathrm{~s}$ exposure time, which gave a cadence of about $47 \mathrm{~s}$. The start and end time of the observations were HJD_UTC 2455547.913994473 and 2455548.085096728, respectively. The time-series data were preprocessed using the standard ARI pipeline and aperture photometry was performed using IRAF/DAOphot.

\subsection{James Gregory Telescope Photometry}

A partial transit was obtained in photometric conditions on 2008 November 30 with a $1 \mathrm{k} \times 1 \mathrm{k}$ CCD detector mounted on the JGT, a $0.95 \mathrm{~m}$ Schmidt-Cassegrain telescope at the University of St. Andrews. The full FOV of the detector is $17^{\prime}$ with a 1 arcsec pixel $^{-1}$ platescale, however vignetting
Table 3

Transit Mid-times

\begin{tabular}{lccr}
\hline \hline$T_{\text {mid }}(" \mathrm{O} ")$ & $\sigma\left(T_{\text {mid }}\right)$ & " $O-C "$ & \multicolumn{1}{c}{ Source } \\
(HJD-2,450,000) & $(\mathrm{s})$ & $(\mathrm{s})$ & \\
\hline 4515.52455 & 11.32 & 5.65793 & LT $(Z)$ \\
4801.47593 & 35.83 & -98.07082 & JGT $(R)$ \\
5123.44587 & 60.35 & -60.35127 & PIRATE $(R)$ \\
5147.45781 & 77.33 & -3.77195 & PIRATE $(R)$ \\
5148.55083 & 113.16 & 133.90439 & PIRATE $(R)$ \\
5158.37107 & 49.04 & -86.75496 & PIRATE $(R)$ \\
5159.46575 & 52.81 & 194.25566 & PIRATE $(R)$ \\
5548.00980 & 20.75 & 3.77195 & FTN $(B)$ \\
5575.29570 & 54.69 & 33.94759 & PIRATE $(R)$ \\
\hline
\end{tabular}

and aberrations create an effective FOV for photometry of approximately $10^{\prime}$. Cousins $R$-band exposures of $90 \mathrm{~s}$ duration were taken over $3 \mathrm{hr}$ from HJD_UTC $=2454801.388296$ to 2454801.517059 , with a total of 104 photometric points. Standard image processing, source detection, and aperture photometry were performed using the Cambridge Astronomical Survey Unit catalog extraction software (Irwin \& Lewis 2001). The software has been compared with SExtractor and found to be very similar in the completeness, astrometry, and photometry tests. We adopted an 8 pixel radius aperture to match the typical seeing for the night $\left(\right.$ FWHM $\left.\sim 8^{\prime \prime}\right)$. Differential photometry was generated from the instrumental magnitudes using the combined flux of seven nearby stars of similar brightness to WASP-12.

\section{RESULTS AND INTERPRETATION}

\subsection{The Orbital Ephemeris}

The elapsed time between the WASP-12 b discovery paper photometry (Hebb et al. 2009) and our two HST visits was 18 months and 2 years, respectively. Since we anticipated that the interpretation of our NUV time-series data could be critically dependent on the WASP-12 orbital ephemeris, we reexamined it. We took our PIRATE, FTN, and JGT data, the Liverpool Telescope $Z$ band from Hebb et al. (2009), together with all available SuperWASP data, and determined a new linear ephemeris using the MCMC fitting routine of Collier Cameron et al. (2007), updated by Enoch et al. (2010), as used by the SuperWASP consortium. We obtained a new ephemeris of

$$
\begin{aligned}
T_{\text {mid }}(\mathrm{HJD})= & 2454852.7739_{-0.00014}^{+0.00014} \\
& +N \times 1.09142206_{-0.000000031}^{+0.00000033}
\end{aligned}
$$

Excluding the SuperWASP data and those observations that did not capture any part of the transit, we then examined the data for transit timing variations (TTVs). We phase-folded the 15 remaining light curves and renormalized each observation against a model light curve appropriate to its color using $\chi^{2}$ minimization. A phase offset from Equation (1) was determined by $\chi^{2}$ minimization for each individual light curve. These offsets define the observed ("O") mid-transit time for each observation, and the deviations (" $\mathrm{O}-\mathrm{C}$ ") from the mid-transit time calculated from Equation (1) (" $C$ ") are tabulated in Table 3. Figure 3 shows our PIRATE data phase-folded on our new ephemeris, both with and without the "O-C" corrections.

Our linear ephemeris is well constrained at either end by precise measurements from Liverpool Telescope and the Faulkes Telescope North. Maciejewski et al. (2011) observed two transits and suggested their measurements might constitute TTV 


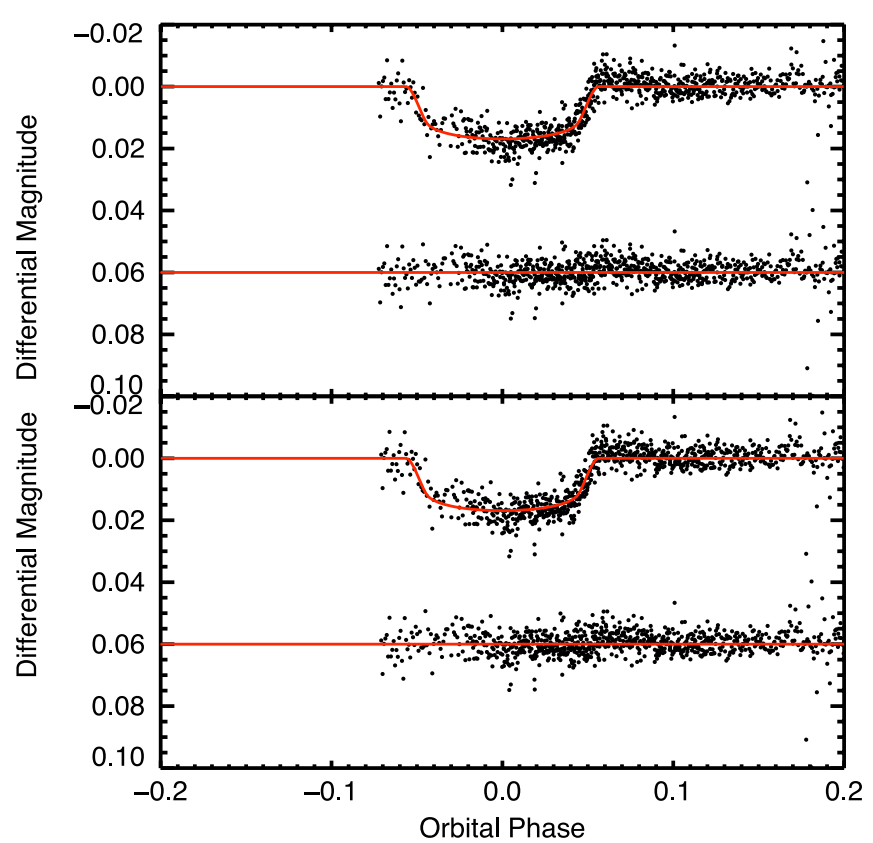

Figure 3. PIRATE $R$-band data folded about our linear ephemeris (Equation (1)); the residuals after subtracting the $R$-band model from the SuperWASP MCMC run (red line) are shown offset. Top panel: with phase-offsets corresponding to the $O-C$ values applied to the data. Bottom panel: without the phase-offsets applied.

(A color version of this figure is available in the online journal.)

detections. We note that our new ephemeris is consistent with the Chan et al. (2011) transit timings and that we suspect the error estimates of Maciejewski et al. (2011) are rather optimistic. Other light curves gathered with comparable facilities lead to larger timing uncertainties.

None of the PIRATE or JGT observations captured complete transits, which is one reason why they have large timing uncertainties. We would not claim a detection of TTVs/TDVs based on these data alone. WASP-12 b may exhibit TTVs with amplitudes of $\sim 200$ s. Further high quality light curves are needed to address this hypothesis.

\subsection{NUV Spectroscopic Coverage}

Figure 1 shows the grand total flux-calibrated spectra for our two HST visits. The Visit 2 spectral coverage is redward of the Visit 1 coverage, and the two ranges overlap for about half of the total coverage. As noted in Section 2.1, the Visit 2 spectra have slightly lower fluxes, largely due to the (uncalibrated) decline in COS throughput. Both spectra are radial velocity $(\mathrm{RV})$ corrected to the WASP-12 rest frame by line profile fitting to the two spectra, thus determining an empirical wavelength offset between the two visits. This correction was applied before generating Figure 1 and subsequent figures.

Figure 1 also shows the stellar continuum level (dashed line). There is prodigious stellar photospheric absorption in WASP-12's atmosphere throughout; no point in our NUV spectra is consistent with unabsorbed continuum. Figure 1 shows synthetic fluxes calculated using the LLMODELS stellar model atmosphere code (Shulyak et al. 2004), assuming the fundamental parameters, metallicity, and detailed abundance pattern given by Fossati et al. (2010a). LLMODELs assumes local thermodynamical equilibrium for all calculations, adopts a plane-parallel geometry, and uses direct sampling of the line opacity allowing the computation of model atmospheres with
Table 4

Simple Transit Model Fits to NUV Light Curves

\begin{tabular}{lccccc}
\hline \hline Band & $\begin{array}{c}\text { Visit } \\
(\mathrm{s})\end{array}$ & $\chi_{v}^{2}$ & $\begin{array}{c}N_{\text {params }} \\
N_{\text {data }}\end{array}$ & $R / R_{*}$ & $\Delta \phi$ \\
\hline A & 1 & 0.276 & 2 & 0.189 & -0.0279 \\
B & 1 & 0.864 & 2 & 0.146 & -0.0186 \\
C & 1 & 0.276 & 2 & 0.186 & -0.0170 \\
A & 2 & 7.77 & 2 & 0.118 & -0.124 \\
B & 2 & 4.11 & 2 & 0.113 & 0.0050 \\
C & 2 & 8.32 & 2 & 0.136 & -0.1271 \\
A & $2 \mathrm{NF}$ & 11.5 & 1 & 0.237 & -0.0475 \\
B & $2 \mathrm{NF}$ & 8.22 & 1 & 0.116 & -0.0204 \\
$\mathrm{C}$ & $2 \mathrm{NF}$ & 15.8 & 1 & 0.224 & -0.0499 \\
AO & 1,2 & 4.002 & 6 & 0.147 & -0.0241 \\
BO & 1,2 & 1.823 & 6 & 0.129 & 0.0034 \\
CO & 1,2 & 4.74 & 6 & 0.122 & 0.0198 \\
AO & $1,2 \mathrm{NF}$ & 4.38 & 5 & 0.1633 & -0.0268 \\
BO & $1,2 \mathrm{NF}$ & 1.25 & 5 & 0.1415 & -0.0159 \\
CO & $1,2 \mathrm{NF}$ & 3.53 & 5 & 0.1424 & -0.0368 \\
\hline
\end{tabular}

Notes. The free parameters are the phase offset from the optical ephemeris, $\Delta \phi$, and the radius, $R$, of a circular occulting disk, expressed as a fraction of the stellar radius. We constrained our search to $R / R_{*} \geqslant 0.1119=R_{\mathrm{P}} / R_{*}$.

individualized (not scaled to solar) abundance patterns. We used the VALD database (Piskunov et al. 1995; Kupka et al. 1999; Ryabchikova et al. 1999) for the atomic line parameters. The resolution applied to the synthetic fluxes matches that of COS, but we did not model the peculiar line-spread function (LSF) of $H S T .^{12}$ The differences between the synthetic and observed spectra are predominantly caused by the HST LSF.

All three regions are affected throughout by many blended photospheric absorption lines. We observe no unabsorbed stellar continuum. The NUVA region is strongly absorbed by many overlapping lines, with lines of $\mathrm{Mg}$ I and Fe I predominating; the NUVB region for both visits is closest to the continuum; while the NUVC region is dominated by absorption in the broad wings of the strong Mg II doublet at $2795.5 \AA$ and $2802.7 \AA$.

\subsection{NUV Transit Light Curve}

In Paper I, we observed an NUV transit that was significantly deeper than the optical transit, and which began with an early ingress compared to the optical ephemeris. These conclusions from our Visit 1 data alone are strengthened by the re-reduction described in Section 2.1. We quantify this by fitting the simplest possible transit model, assuming a circular occulting disk transiting a uniform brightness (no limb-darkening) star. We fix all the parameters of this model to the values demanded by WASP-12 b's optical transit, except for two free parameters: $R / R_{*} \geqslant R_{\mathrm{P}} / R_{*}$ and $\Delta \phi$, describing the radius and phase offset of the NUV occulting disk compared to the transit of the optically opaque planet with $R=R_{\mathrm{P}}$ and $\Delta \phi=0$ across the star of radius $R_{*}$. The normalization of the data to the model is also a free parameter. We report our model fitting in Table 4, using the reduced $-\chi^{2}$ statistic, $\chi_{v}^{2}$, which is the appropriate statistic for assessing the goodness of fit. For completeness, we also report the number of degrees of freedom, i.e., the number of parameters including normalization minus the number of fitted data points. The results for Visit 1 shown in Figure 4 and at the top of Figure 5 and Table 4 are consistent with Paper I: for NUVA, NUVB, and NUVC the best-fit transits are early by 43 minutes,

12 http://www.stsci.edu/hst/cos/documents/newsletters/full_stories/ 2010_02/1002_cos_lsf.html 


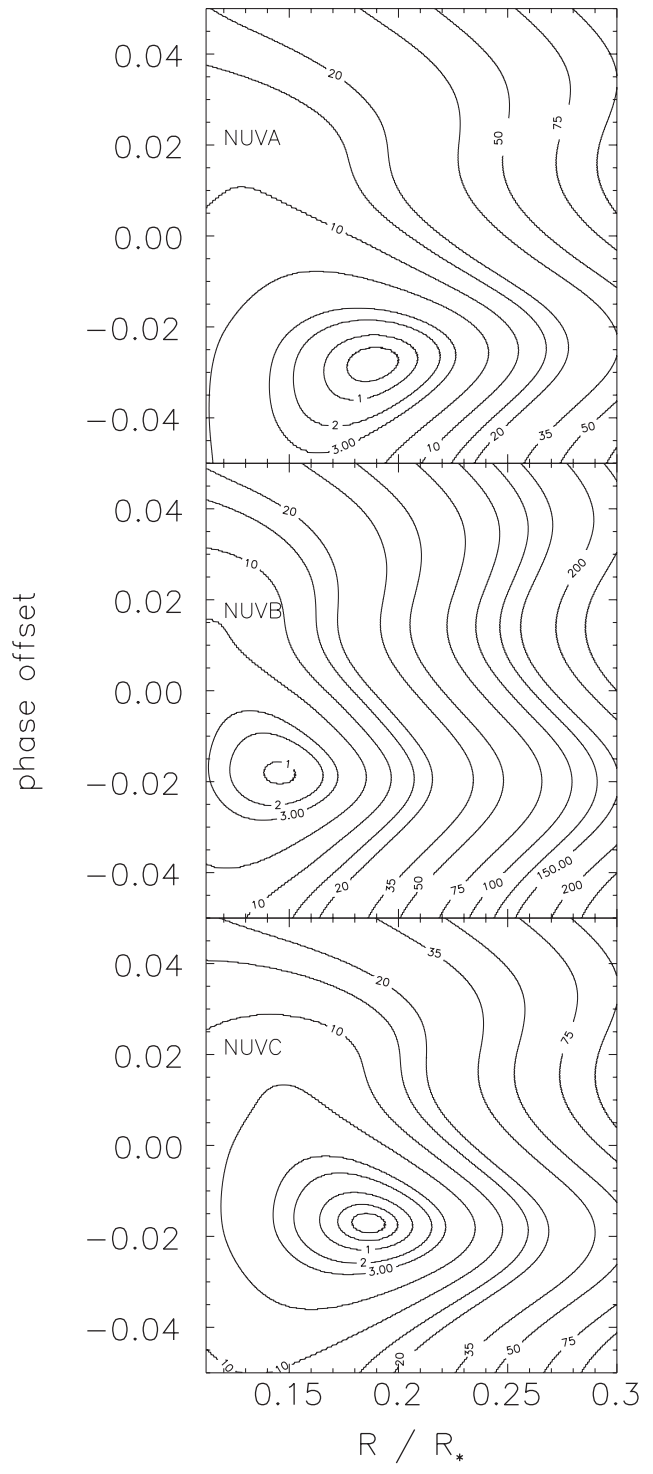

Figure 4. $\chi_{v}^{2}$ grids from fitting model transits to our Visit 1 NUV data. We model circular opaque disks transiting a uniform brightness stellar disk. By allowing the normalization of the model light curves to vary, we find the best-fit transit for each value of phase and radius ratio without making any assumptions about which of the empirical points represent out-of-transit coverage. All three light curves are best fit by transits that occur early, and the best-fit models in all cases correspond to disks much larger than that of the optical planet: $R_{\mathrm{P}} / R_{*}=0.1119$, which is the smallest value of $R / R_{*}$ shown.

29 minutes, and 26 minutes, respectively. As Table 4 shows, the best-fit radii for the occulting disk are significantly larger than that of the optically opaque planet $\left(R_{\mathrm{P}} / R_{*}=0.1119\right.$; Chan et al. 2011) with an enhancement in radius of $70 \%$, i.e., almost a factor of three in area, for the NUVA and NUVC bands. The NUVA and NUVC bands provide extremely sensitive probes for the presence of low density gas at temperatures of several thousand degrees because these bands contain $\sim 10^{3}$ overlapping spectral lines. We discuss the spectral information in detail in Section 3.4 below. We do not imagine that the low density gas we have detected surrounding and preceding WASP-12 b actually presents a circular cross-section to us during its transit; this was the simplest model we could imagine, and we have few data points to fit. As the upper panels in Figure 5 show, with two free parameters (three including normalization) the model is already able to produce an improbably good fit to the data and this suggests that our error bars (derived from photon-counting statistics) are not underestimated.

Visit 2 was timed to provide coverage of the orbital phases missing due to Earth-occultation in our Visit 1 data. The two visits provide well-sampled coverage between $-0.17<$ $\phi_{\text {orb }}<0.12$. Unfortunately, the Visit 2 data defy straightforward interpretation. The lower panels of Figure 5 show the Visit 2 data along with the best-fit simple transit models to the three NUV bands. As the corresponding entries in Table 4 show, none of these fits are formally acceptable, with $\chi_{v}^{2}$ significantly above one in all cases. The NUVB band produces the best-fit transit for Visit 2, but this fit essentially reproduces the transit of the optically opaque planet (dotted line in Figure 5), with points before and after the transit deviating significantly from the model. The best-fit NUVB model is slightly late compared to the optical transit because the Visit 2 point that samples optical ingress is higher than the optical transit would predict. The Visit 2 NUVA and NUVC light curves strongly resemble each other. Throughout, the point-to-point deviations in the two light curves appear similar. In both cases, the Visit 2 data are strongly absorbed before optical first contact, with the point sampling optical ingress lying above the OOT level, while the Visit 1 data appear more strongly absorbed between optical second and third contacts. The best-fitting model for the Visit 2 NUVA and NUVC light curves is a transit that occurs so early that it fails to overlap the optical transit. This is clearly unphysical: the planet is surely opaque to NUV light. These fits result from the anomalously high third data point (arising from the third HST orbit) in the Visit 2 NUVA and NUVC light curves: in both cases, the exposure spanning the optical ingress is the secondhighest in the light curve. Our simple model requires a strict relationship between the depth and duration of the fitted transit. Since the "orbit 3 point" is high, the model places it above the OOT level and as the pre-optical-ingress NUVA and NUVC data points are both lower than the fitted OOT level, the best-fit transit model places the entire transit early.

The aberrantly high Visit 2 orbit 3 data point in the NUVA and NUVC light curves thus defies the simple explanation we developed from Visit 1. We can think of three plausible explanations for this: (1) these are noisy data and this simply happens to be a point with a positive deviation; (2) there was a short-lived stellar flare during Visit 2 orbit 3; (3) the gas which absorbs before first contact is clumpy, and there was a clear window to the star during Visit 2 orbit 3. Vidotto et al.'s (2011) bow shock model can produce such a "window" configuration. The same pattern independently appearing in both the NUVA and NUVC light curves makes the first explanation far less likely than it would otherwise be, and our fits to the Visit 1 light curve suggest our error estimations are ample. We will discuss the stellar flare hypothesis in Sections 3.6 and 4.1, and the window hypothesis in Section 4.1.

The Visit 2 data with the aberrant third-orbit data masked out produces the fits shown in Figure 6 and Table 4 (entries for visit "2NF"). The NUVB fit now has a slightly deeper transit that occurs earlier because the fit has placed the orbit 4 point to span the beginning of egress. This fit, and the adjustments to it we might make if we relax our arbitrary assumption that the extended gas presents a circular cross-section, is consistent with the interpretation we made in Paper I: the absorbing gas creates a larger total absorbing area than inferred from the optical transit, and its ingress precedes that of the optically opaque planet. The NUVA and NUVC "best" fits are comical. Because the orbit 1, 2 , and 4 points in both cases lie so far below the orbit 5 point, 


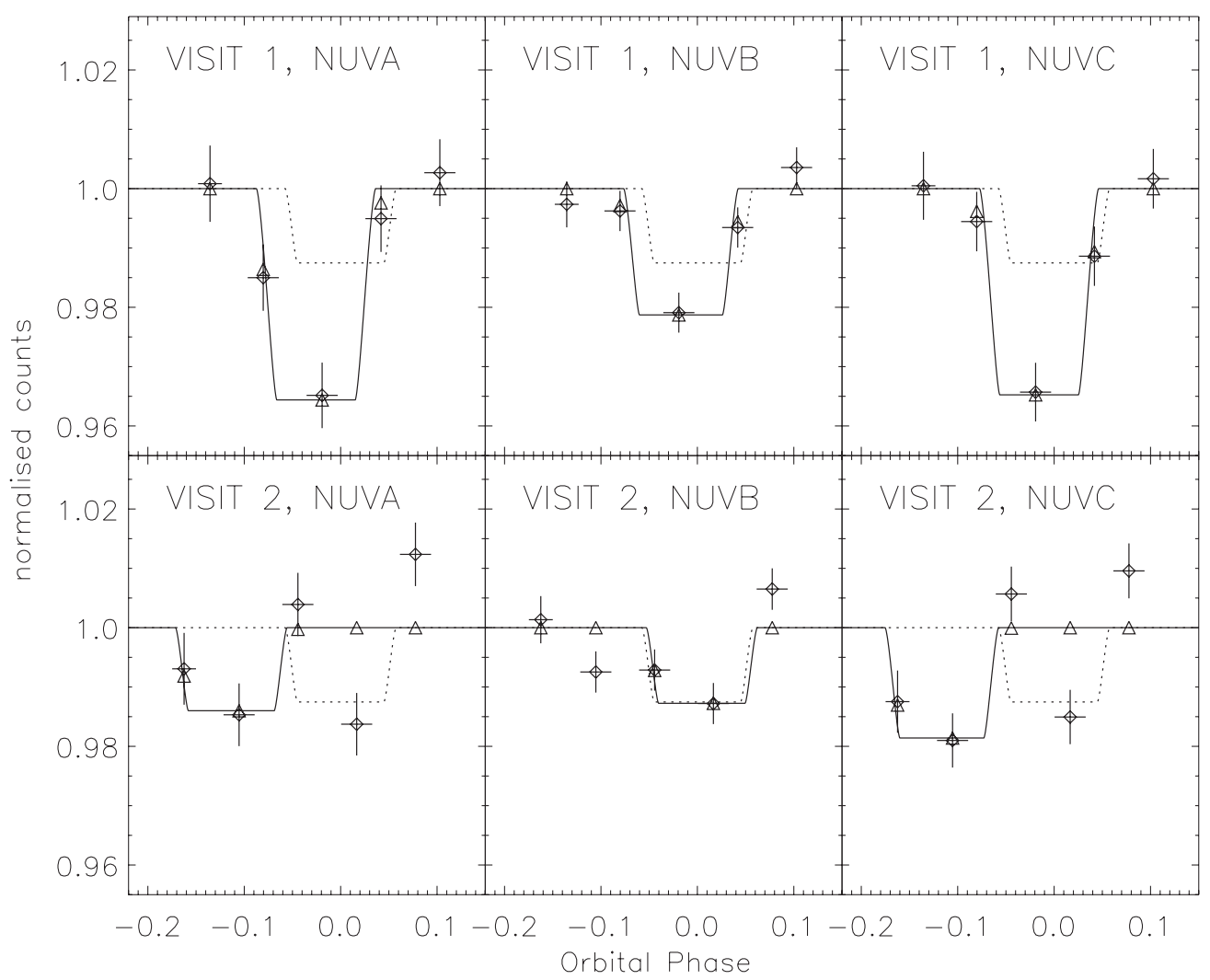

Figure 5. Best-fitting simple transit models (solid lines) for each of our NUV light curves (diamonds). Triangles show the integral of the best-fit model over the exposure times used. In Visit 1, we obtain good fits which reveal an NUV transit deeper than the optical transit and an early ingress. Visit 2 is less clear-cut: the 3rd data point in all three bands is high compared to the models fitting Visit 1, particularly for NUVA and NUVC. For these two bands, the best-fit model places this anomalously high point after egress so the "best-fit" transit does not overlap the optical transit (dotted line).

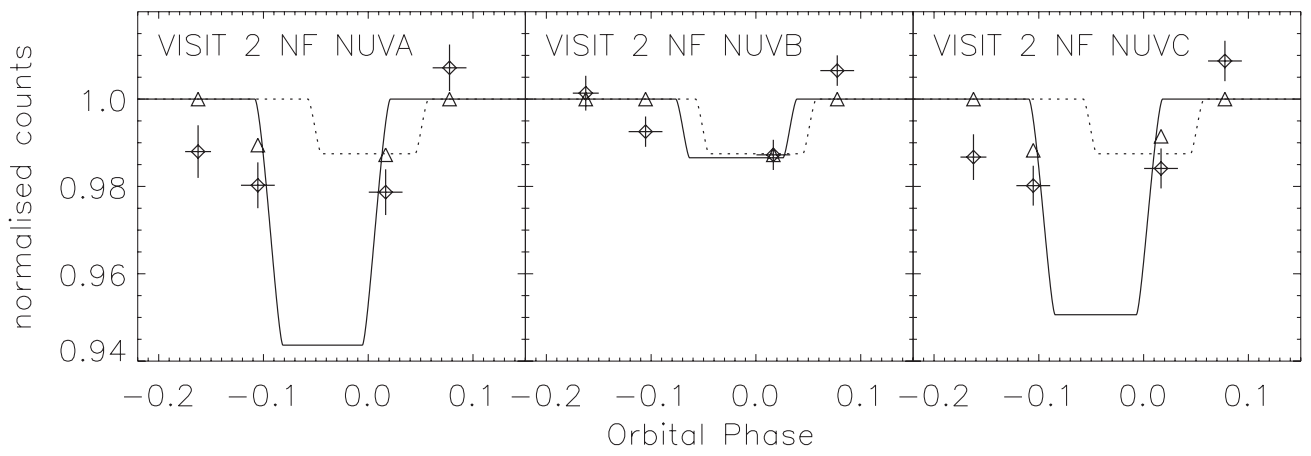

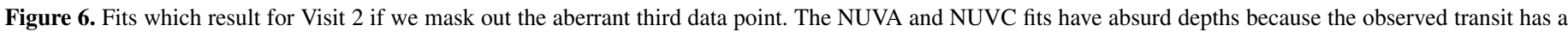
long duration and we fit a circular occulter. A more plausible model would have absorbing gas extended in the orbital plane. Symbols as in Figure 5.

the relationship between transit depth and duration favors fits in which the transit depth is much deeper than any data point. A more sensible interpretation would be that the absorbing gas is extended along the orbital plane and presents a significantly non-circular cross-section, so the duration is longer than that of the models while the depth is shallower.

The fits we have performed separately on the two individual visits suggest that the configuration of the absorbing gas changed between the two visits. Indeed Vidotto et al. (2011) interpret this variability. Nonetheless, for completeness, we now proceed to examine the data from the two visits together.

Figure 7 interleaves the two visits using only the photons detected within the overlapping wavelength regions of the RVcorrected spectra. It is immediately apparent that the Visit 1 data (black circles) lies above the Visit 2 data (red triangles). We attribute this to the known declining throughput of the
COS/G285M configuration (Osten et al. 2011). We corrected for this by boosting the Visit 2 count rates by $5.52 \%$ using an estimate from Osten et al. (2010), but the rate of sensitivity decline is not precisely known for our instrument configurations. The "corrected" Visit 2 data are shown as blue squares.

The background level differed between the two visits, with roughly twice as many background counts during Visit 2. The temporal behavior of the background also differed, with a gradual increase in background during Visit 1 contrasting with a decrease in background during Visit 2. We corrected for background, so this should not affect our light curves. The corrected Visit 2 data appear to match well at phases following the transit for NUVA and NUVC, but the corrected NUVB light curve from Visit 2 appears systematically lower than the Visit 1 light curve. The Visit 2 orbit 3 point remains aberrant in NUVC, but restricting the wavelength coverage to only the overlapping 


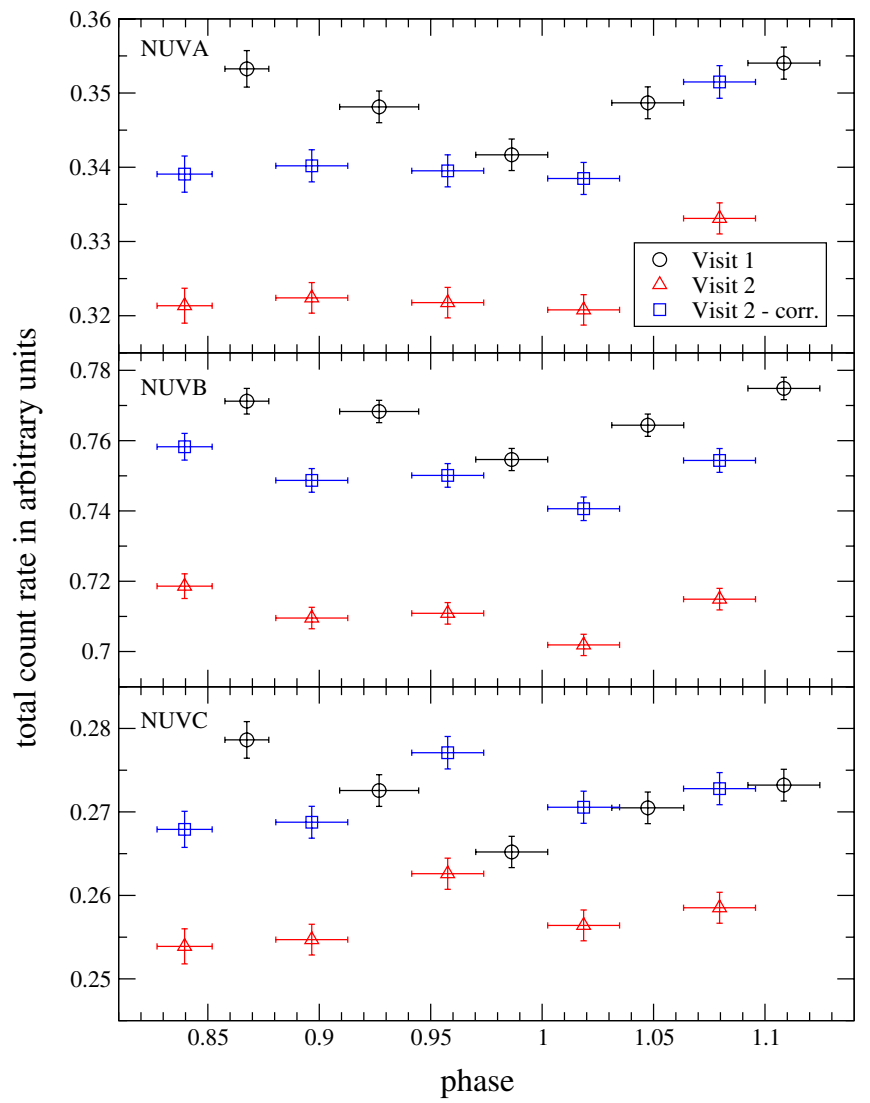

Figure 7. Count rates from the NUVA, NUVB, and NUVC overlapping spectral regions in the two HST visits. Circles show Visit 1, triangles show Visit 2, and squares show Visit 2 after multiplying by 1.052 to correct for the decline in instrument sensitivity between the two visits.

(A color version of this figure is available in the online journal.)

region has dramatically lowered the NUVA orbit 3 point. We will return to discuss the reasons for this in Section 4.1.

Our NUV light curves, particularly the NUVA and NUVC bands, suggest that in Visit 2 perhaps the absorbing gas obscured the stellar disk at phases as early as $\phi_{\text {orb }}=0.83$, in which case Visit 2 contains no genuine pre-transit data. Figure 8 shows the light curves produced using all the data (i.e., not just the overlapping wavelength regions), arbitrarily setting the last point in each visit to (an OOT level of) 1.0. The NUVB light curve now looks perfectly consistent with the interpretation we made in Paper I: the transit is deeper than the optical transit and appears to be preceded by absorption occurring before optical ingress. The NUVA and NUVC light curves also look reasonably consistent with this interpretation, with much more significant pre-ingress absorption occurring in Visit 2.

We performed simple transit fits (as described above) to the combined data from the overlapping wavelength regions in the two visits, allowing the normalization of each visit to vary independently. These fits thus have four free parameters in total: $R, \Delta \phi$, and two independent normalization values. Figure 9 shows the best-fitting models, along with the data after applying the best-fit normalizations. As Table 4 quantifies (entries for bands AO, BO, CO; Visits "1, 2"), none of the three fits in Figure 9 are formally acceptable. Nonetheless, the NUVA and NUVB fits are broadly consistent with our interpretation in Paper I. The best-fit transit is significantly deeper than the optical transit and the light curves show signs of absorption from diffuse gas before optical ingress. The NUVC band produces formally

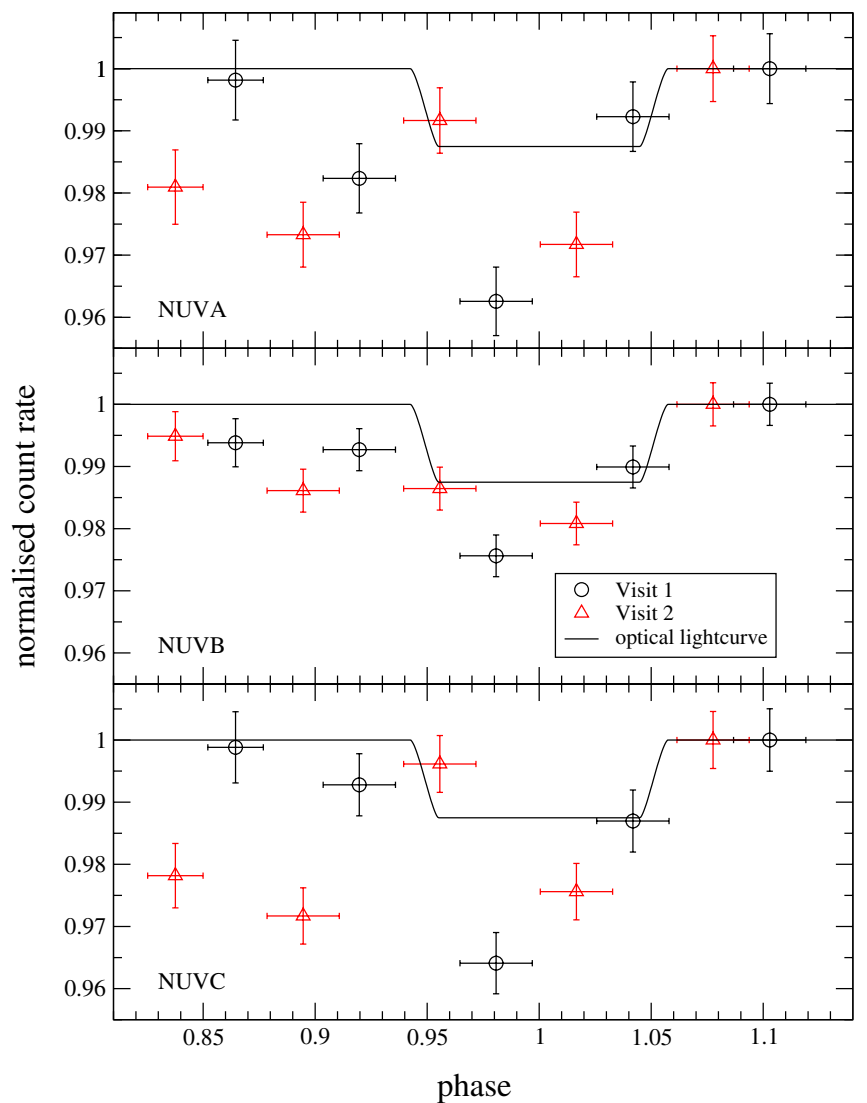

Figure 8. Light curves from all of our HST data, with each HST visit normalized to unity in the last $H S T$ orbit. Note that the wavelength regions contributing to the two visits differ, as indicated in Figure 1.

(A color version of this figure is available in the online journal.)

the worst fit, and the first half of the light curve shows enormous scatter between the sets of points from the two visits. The best-fit model transit is late compared to the optical transit: a fit which moved the ingress to after the anomalously high Visit 2 orbit 3 point has been preferred.

Figure 10 shows the best fits to the interleaved data without the Visit 2 orbit 3 point. These fits (entries for Visits “1, 2NF") are all broadly consistent with our Paper I conclusions. For the NUVB and NUVC bands, the best $\chi_{v}^{2}$ is better than the fits shown in Figure 9.

In both NUVA and NUVC, as Figure 1 shows, the wavelength ranges covered only by the Visit 1 data are strongly absorbed by the stellar photosphere, while the wavelength ranges covered only by the Visit 2 data are significantly less strongly absorbed. If the diffuse absorbing gas has broadly the same ionic composition (i.e., abundance mix and temperature) as the stellar photosphere, then this would predict a deeper transit in Visit 1 NUVA and NUVC than in Visit 2 NUVA and NUVC. This is exactly what Figure 5 and Table 4 show.

Figure 1 shows that the NUVB spectral region, for both visits, is the closest to the stellar continuum: there is relatively little photospheric absorption, and we might similarly expect relatively little absorption from the extended cloud of diffuse gas. Of course, without knowing the composition and physical conditions of this gas, it is impossible to assert that this is definitely true, but it is the simplest assumption one could make a priori. Our NUVB data suggest a deeper transit than that of the optical light curve, but of the three spectral regions it is the closest to the optical curve. Note that the NUVB data have the 


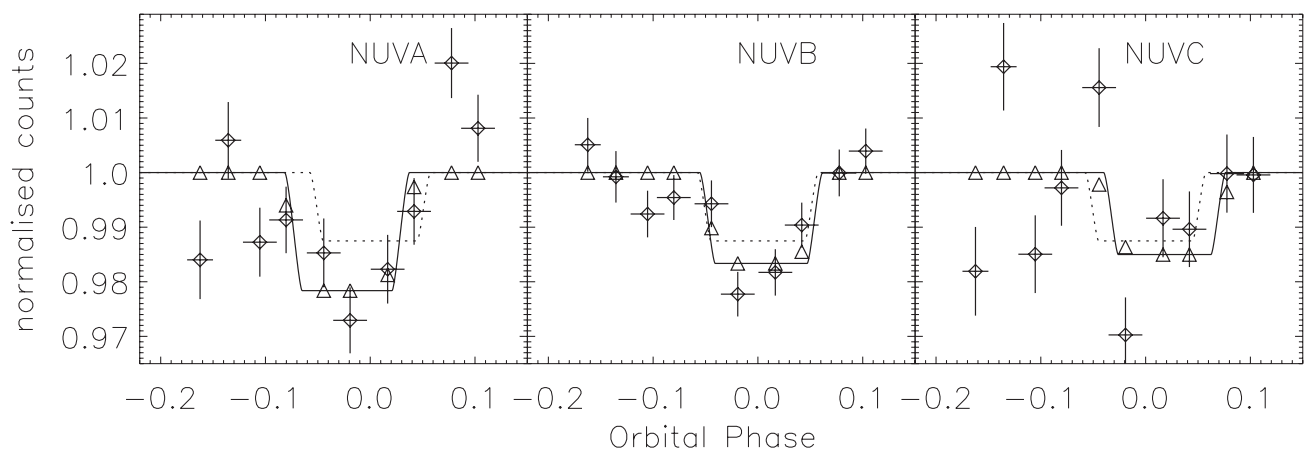

Figure 9. Best-fitting simple transit models for our interleaved NUV data. None of the fits are formally acceptable, but the NUVA and NUVB results are broadly consistent with a deeper transit than the optical and absorption from diffuse gas which precedes the optical transit (dotted line). The best-fit NUVC transit occurs late, but the first half of the interleaved light curve has extremely high scatter so this fit should clearly not be taken at face value. Symbols are as in Figure 5.

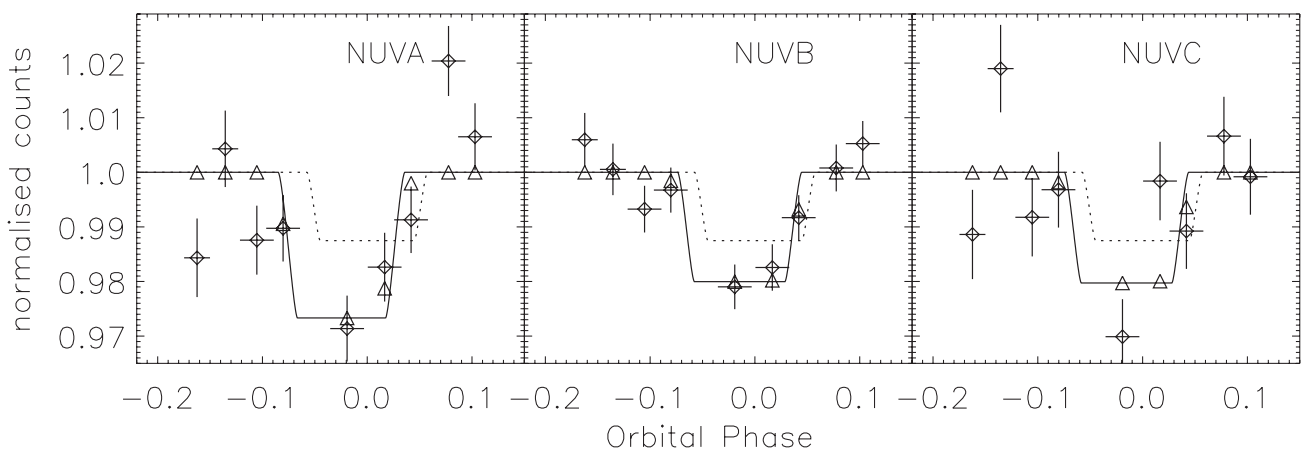

Figure 10. Best-fitting simple transit models for our interleaved NUV data with the Visit 2 Orbit 3 point masked out. None of the fits are formally acceptable, but the results are broadly consistent with a deeper transit than the optical and absorption from gas preceding optical transit (dotted line). Symbols are as in Figure 5 .

smallest uncertainties because, being relatively unabsorbed, we detect most photons from the star in NUVB.

Each (version of the) NUVB light curve (Figures 5-10) shows indications of an early ingress in NUVB. Our NUVA and NUVC light curves are also broadly consistent with this, except for the anomalous count rates observed in Visit 2 orbit 3 . This aberrant point appears in both NUVA and NUVC when we considered the entire wavelength range (Figures 5 and 8), but only in NUVC when we extract light curves from the overlapping wavelength ranges (Figures 7 and 9). The Visit 2 orbit 3 NUVA point shifts downward by $4 \sigma$ when we restrict the wavelength range. We think this is significant and will return to discuss the implications of this piece of evidence in Section 4.1.

In summary, the NUV light curves are broadly consistent with our principal conclusions in Paper I. The transit is deeper than in the optical and is preceded by an early ingress, though the point for the third orbit in Visit 2 deviates from this description when some wavelength regions are considered. There is clearly NUV absorption occurring before optical first contact.

\subsection{The Wavelength Resolved Transit}

The enhanced transit depths seen in Figures 4, 5, 6, 8, 9, and 10 result from summing over the $\sim 40 \AA$ covered in each of our NUVA, NUVB, and NUVC spectra (or the $\sim 30 \AA$ of overlapping wavelength coverage for the last two figures listed). The enhancement of the NUV transit depth compared to the optical transit depth is due to absorption in the diffuse gas surrounding the planet. This absorption is caused by bound-bound transitions in atomic and ionic species. A priori we do not know which species are present in this gas, but we do know that there are thousands of overlapping spectral lines within the wavelength ranges we have observed. Since we have time-series spectral data, we can examine the transit at any spectral resolution greater than or equal to that of the data. In Paper I, we presented evidence for detections of enhanced transit depths in $\mathrm{Mg}$ II and other lines of neutral and ionized metals. With the addition of Visit 2, we have extended our wavelength coverage, our temporal coverage, and increased the $\mathrm{S} / \mathrm{N}$ of the overlapping wavelength region, so we examined our Visit 1 and Visit 2 data afresh for evidence of enhanced transit depths at particular wavelengths.

We used the methods of Paper I to detect wavelengths with significantly over-deep transits. In Paper I, we used the average of Visit 1 orbits 1 and 5 for the OOT spectrum and orbit 3 for the in transit (IN) spectrum. As Figures 2 and 5 demonstrate, there is no reason to change this, so we used the same portions of data (re-reduced as detailed in Section 2.1). Visit 2 shows no clear pre-ingress OOT data, so in this case we used orbit 5 for the OOT spectrum and orbit 4 for the IN spectrum. Consequently, the propagated error estimate for our Visit 2 OOT spectrum is larger that that for the Visit 1 OOT spectrum.

For each visit, we define the ratio spectrum, $d_{i}(=\mathrm{IN} / \mathrm{OOT})$, where IN is the in-transit flux and OOT is the out-of-transit flux, to measure the transit depth at wavelength $\lambda_{i}$. We examine $d_{i}$ to determine wavelengths $\lambda_{i}$ with statistically significant excess transit depths. These are the wavelengths where the diffuse gas absorbs. These individual contributions combine to produce the excess depth in the light curves examined in Section 3.3. We report the wavelengths of these points in Table 5 and in Figure 11. In Table 5, we use two different criteria to assess anomalously low points, i.e., those deviating by $3 \sigma$, as we did in Paper I. The first of these examines the ratio spectrum against the error estimate we obtain from the propagated (Poissondominated) errors in the reduction. We label this error estimate as $\left.\sigma d_{i}\right|_{\text {prop }}$, and report in Table 5 wavelengths where the ratio 
Table 5

Wavelength of the Visit 1 and Visit 2 Spectral Points Deviating More than $3 \sigma$, Adopting Two Different $\sigma \mathrm{s}: \sigma_{d_{\lambda} \mid \operatorname{lexp}}$ and $\sigma_{d_{\lambda} \mid \text { prop }}$

\begin{tabular}{|c|c|c|c|c|c|c|c|c|c|c|c|}
\hline $\begin{array}{l}\text { Wavelength } \\
\lambda-2000 \AA \\
\text { Visit } 1 \\
3 \times \sigma_{d_{i} \mid \exp }\end{array}$ & $\begin{array}{c}\text { Wavelength } \\
\lambda-2000 \AA \\
\text { Visit } 2 \\
3 \times \sigma_{d_{i} \operatorname{lexp}} \\
\quad \mathrm{NU}\end{array}$ & $\begin{array}{l}\text { Wavelength } \\
\lambda-2000 \AA \\
\text { Visit } 1 \\
3 \times \sigma_{\left.d_{i}\right|_{\text {prop }}} \\
\text { VA }\end{array}$ & $\begin{array}{c}\text { Wavelength } \\
\lambda-2000 \AA \\
\text { Visit } 2 \\
3 \times \sigma_{d_{i} \mid \text { prop }}\end{array}$ & $\begin{array}{c}\text { Wavelength } \\
\lambda-2000 \AA \\
\text { Visit } 1 \\
3 \times \sigma_{\left.d_{i}\right|_{\exp }}\end{array}$ & $\begin{array}{c}\text { Wavelength } \\
\lambda-2000 \AA \\
\text { Visit } 2 \\
3 \times \sigma_{d_{i} \mid \exp } \\
\quad \mathrm{NU}\end{array}$ & $\begin{array}{l}\text { Wavelength } \\
\lambda-2000 \AA \\
\text { Visit } 1 \\
3 \times \sigma_{\left.d_{i}\right|_{\text {prop }}} \\
\text { VB }\end{array}$ & $\begin{array}{c}\text { Wavelength } \\
\lambda-2000 \AA \\
\text { Visit } 2 \\
3 \times \sigma_{\left.d_{i}\right|_{\text {prop }}}\end{array}$ & $\begin{array}{c}\text { Wavelength } \\
\lambda-2000 \AA \\
\text { Visit } 1 \\
3 \times \sigma_{d_{i} \mid \exp }\end{array}$ & $\begin{array}{c}\text { Wavelength } \\
\begin{array}{c}\lambda-2000 \AA \\
\text { Visit } 2 \\
3 \times \sigma_{d_{i} \mid \exp } \\
\mathrm{NU}\end{array}\end{array}$ & $\begin{array}{l}\text { Wavelength } \\
\lambda-2000 \AA \\
\text { Visit } 1 \\
3 \times \sigma_{\left.d_{i}\right|_{\text {prop }}} \\
\text { VC }\end{array}$ & $\begin{array}{c}\text { Wavelength } \\
\lambda-2000 \AA \\
\text { Visit } 2 \\
3 \times \sigma_{\left.d_{i}\right|_{\text {prop }}}\end{array}$ \\
\hline 538.484 & & 537.535 & & 661.605 & & 657.054 & & & 792.434 & 776.577 & \\
\hline 539.002 & & 537.880 & & 662.022 & & 664.316 & & 793.963 & & 776.818 & \\
\hline 539.045 & & 538.139 & & 663.649 & & 664.816 & & 794.442 & & 778.785 & \\
\hline 543.442 & & 538.312 & & 666.649 & & 666.691 & & 794.842 & & 779.026 & \\
\hline 546.758 & & 538.916 & & & 667.763 & 666.733 & & 794.922 & & 784.399 & \\
\hline 557.028 & & 538.959 & & & 667.888 & & 667.763 & 795.401 & & 785.040 & \\
\hline 562.344 & & 539.520 & & 669.522 & & & 669.432 & 795.561 & & & 787.704 \\
\hline 562.473 & & 539.606 & & 670.313 & & & 669.474 & 795.641 & & & 787.945 \\
\hline & 563.215 & 540.166 & & & 671.851 & & 672.310 & 797.957 & & & 788.065 \\
\hline 563.501 & & $\begin{array}{l}540.641 \\
540.856\end{array}$ & & & $\begin{array}{l}672.018 \\
\mathbf{6 7 8 . 8 0 7}\end{array}$ & & $\begin{array}{l}678.558 \\
680.804\end{array}$ & $\begin{array}{l}801.428 \\
802.185\end{array}$ & & 788.524 & \\
\hline 575.980 & 575.999 & $\begin{array}{l}540.900 \\
541.072\end{array}$ & & $\begin{array}{l}\mathbf{6 7 8 . 9 5 6} \\
682.895\end{array}$ & ת & 684.179 & 686.995 & $\begin{array}{l}802.425 \\
802.504\end{array}$ & & $\begin{array}{l}791.004 \\
791.084\end{array}$ & 190.902 \\
\hline 576.151 & & 541.158 & & 682.978 & & 687.491 & & 803.023 & & 791.164 & \\
\hline & 576.342 & 541.201 & & 684.759 & & & 687.908 & 803.142 & & & 791.513 \\
\hline & 576.385 & 541.891 & & & 689.360 & 690.056 & & & & 791.604 & \\
\hline & 576.470 & 542.107 & & & 690.936 & 691.089 & & & & & 792.274 \\
\hline & 577.883 & 542.193 & & & 690.978 & & 692.470 & & & & 792.314 \\
\hline 580.925 & & 542.537 & & 695.261 & & 692.618 & & & & & 792.674 \\
\hline & 582.286 & 542.581 & & & 698.681 & & 692.802 & & & 793.123 & \\
\hline & 585.873 & 543.873 & & & 698.805 & 694.601 & & & & & 793.235 \\
\hline & 586.043 & 543.916 & & & 698.847 & 695.261 & & & & 793.243 & \\
\hline & & 544.347 & & & 706.904 & & 695.370 & & & 793.403 & \\
\hline & & 544.390 & & & 710.368 & & 695.784 & & & 793.443 & \\
\hline & & 544.433 & & & & & 697.233 & & & 793.483 & \\
\hline & & 546.155 & & & & & 700.873 & & & & 793.635 \\
\hline & & 546.198 & & & & & 703.146 & & & & 793.715 \\
\hline & & 546.241 & & & & & 706.408 & & & 793.723 & \\
\hline & & 546.715 & & & & & 710.080 & & & & 793.755 \\
\hline & & 547.490 & & & & & 710.203 & & & 793.803 & \\
\hline & & 547.705 & & & & & 710.327 & & & 793.843 & \\
\hline & & 548.952 & & & & & 710.368 & & & 794.003 & \\
\hline & & 549.425 & & & & & & & & 794.083 & \\
\hline & & & 549.902 & & & & & & & & 794.116 \\
\hline & & $\begin{array}{l}\mathbf{5 4 9 . 9 8 4} \\
\mathbf{5 5 0 . 0 2 7}\end{array}$ & & & & & & & & 794.402 & 794.436 \\
\hline & & & 550.248 & & & & & & & 794.522 & \\
\hline & & & 550.291 & & & & & & & & 794.956 \\
\hline & & & 550.550 & & & & & & & & 794.996 \\
\hline & & & 550.636 & & & & & & & & 795.196 \\
\hline & & & 550.680 & & & & & & & & 795.276 \\
\hline & & 550.887 & & & & & & & & & 795.316 \\
\hline & & & 551.629 & & & & & & & & 795.356 \\
\hline & & & 551.758 & & & & & & & & 795.397 \\
\hline & & & 551.802 & & & & & & & & 795.477 \\
\hline & & & 552.708 & & & & & & & 795.481 & \\
\hline & & & 553.010 & & & & & & & & 795.517 \\
\hline & & & 553.139 & & & & & & & 795.521 & \\
\hline & & 553.465 & & & & & & & & & 795.597 \\
\hline & & & 554.131 & & & & & & & 795.601 & \\
\hline & & & 554.691 & & & & & & & & 795.637 \\
\hline & & & 555.424 & & & & & & & 795.721 & \\
\hline & & & 555.510 & & & & & & & 795.920 & \\
\hline & & & 556.975 & & & & & & & 796.080 & \\
\hline & & & 557.277 & & & & & & & 796.120 & \\
\hline & & & 557.578 & & & & & & & 796.160 & \\
\hline & & & 558.310 & & & & & & & 796.200 & \\
\hline & & & 558.396 & & & & & & & 796.280 & \\
\hline & & & 558.870 & & & & & & & & 796.357 \\
\hline & & 558.916 & & & & & & & & 796.479 & \\
\hline & & 559.173 & & & & & & & & & 796.557 \\
\hline
\end{tabular}


Table 5

(Continued)

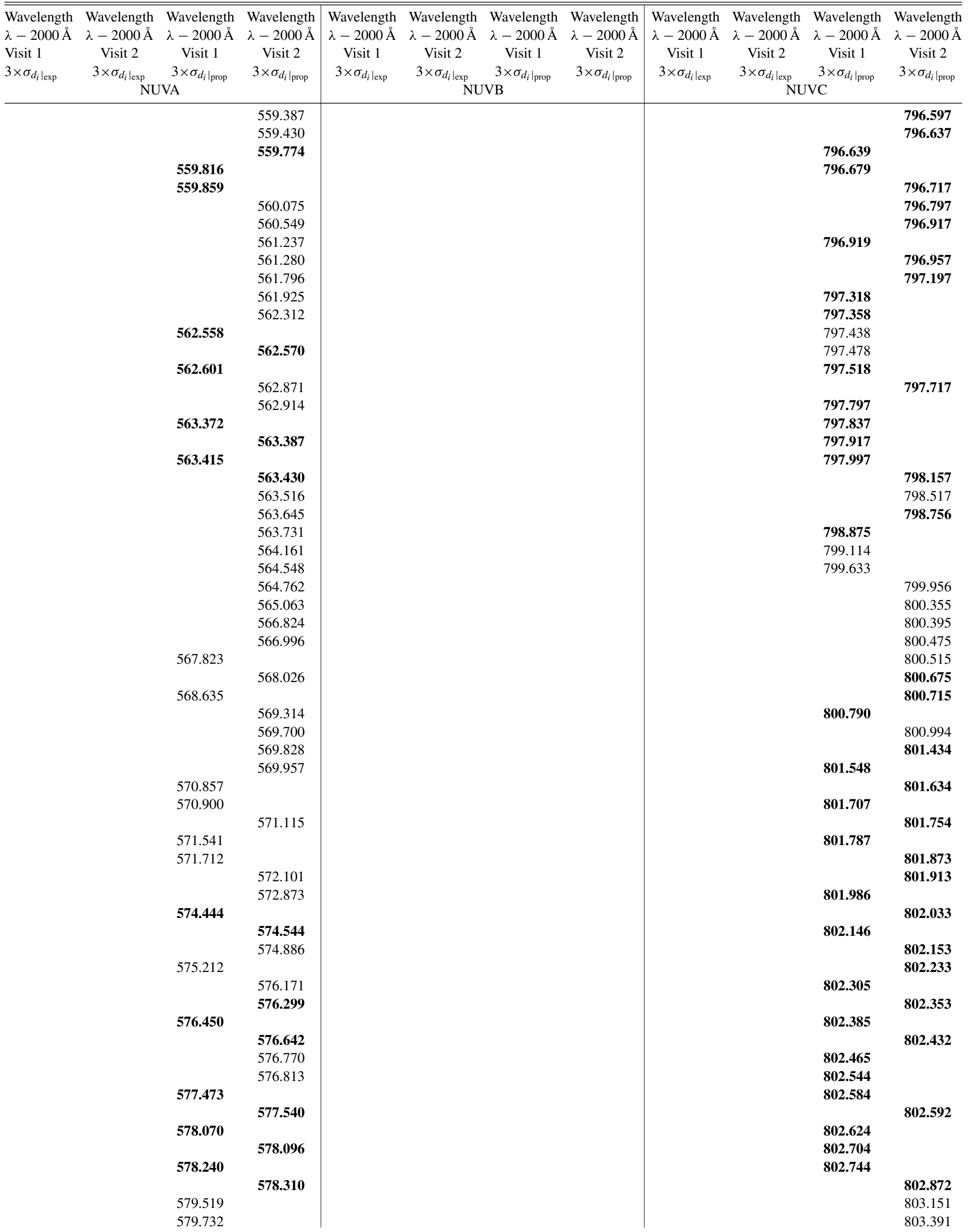


Table 5

(Continued)

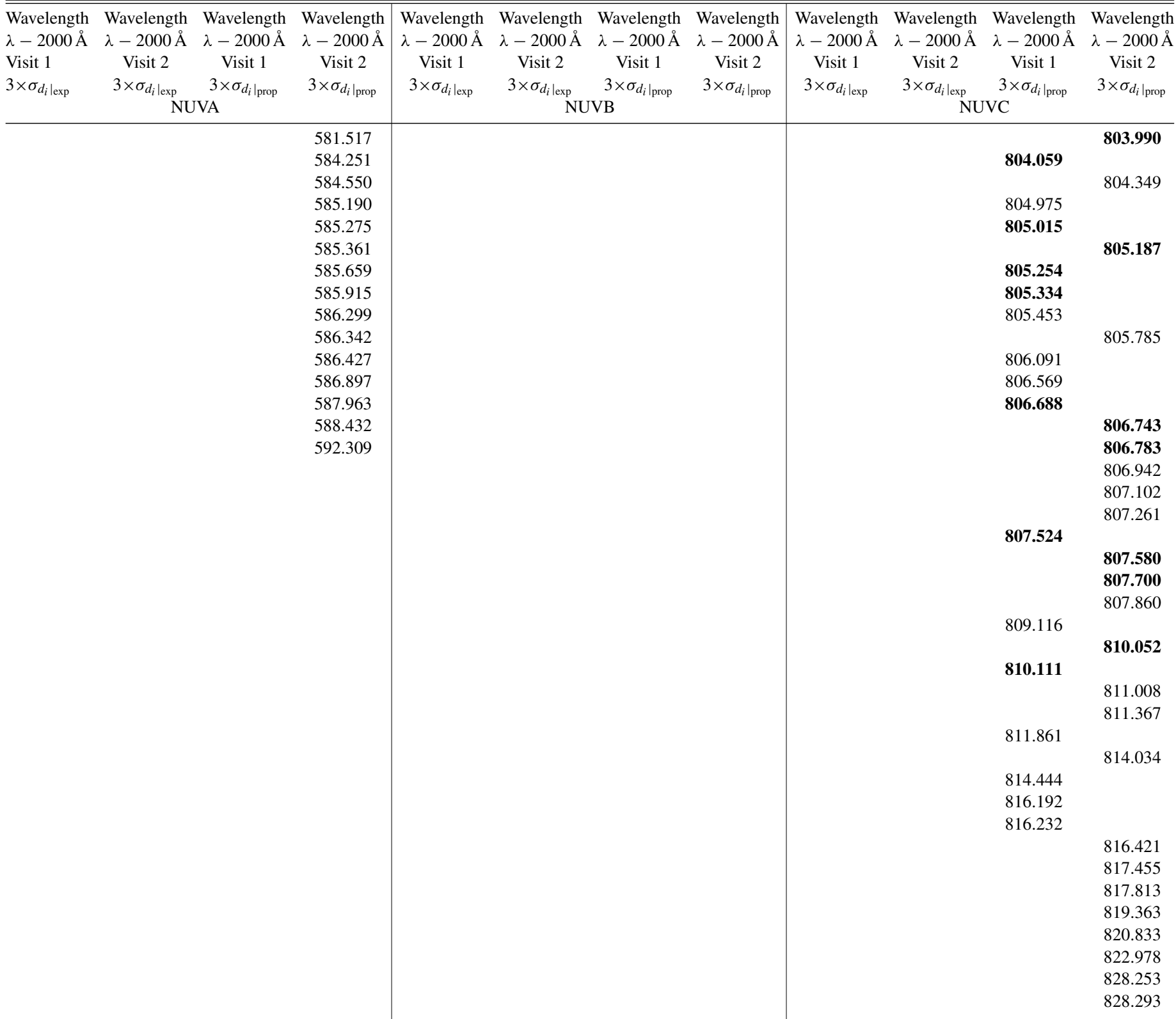

Notes. The deviating points highlighted in bold face are the Visit 2 points that are one resolution element away from a Visit 1 point. All wavelengths are given in Å.

spectrum is $\left.3 \sigma d_{i}\right|_{\text {prop }}$ or more below the median value of the ratio. These wavelengths are also indicated in Figure 11. The second assessment uses the rms scatter, $\sigma_{\left.d_{i}\right|_{\exp }}$, within the ratio spectrum itself in the criterion. As Figure 3 of Paper I showed, the $\sigma_{\left.d_{i}\right|_{\text {exp }}}$ criterion is more likely to pick out points where the signal in the observed stellar spectrum is low.

Our ratio spectra have a total of just under 3100 points, so if the noise is Gaussian distributed, then we expect $\sim 9$ distinct points in each to deviate by more than $\left.3 \sigma d_{i}\right|_{\text {prop. }}$. As Table 5 shows, we find many more deviating points than this. In every case, the points that deviate by more than $\left.3 \sigma d_{i}\right|_{\text {prop }}$ are deviations below the median of the ratio spectrum, indicating extra absorption compared to the median. Where there are positive deviations, these are accompanied by large values of $\left.\sigma d_{i}\right|_{\text {prop }}$ due to the low flux, and hence low S/N of the WASP12 spectrum at that wavelength. Consequently, none of these positive deviations are $\left.3 \sigma d_{i}\right|_{\text {prop }}$. We would expect, nonetheless, to find a small number $(\sim 4 \pm 2)$ of positive deviations due to white noise, but we do not. Possibly our propagated errors are slightly overestimated. This leads us to conclude we have detected statistically significant excess absorption at $\sim 200$ distinct wavelengths in each visit from the gas around WASP-12.

As noted in Section 3.3, the NUVA and NUVC spectral regions are more strongly absorbed within the stellar photosphere than is NUVB. In Section 3.3, we showed that the transit is deeper in wavelength regions where the photospheric absorption is strong. These wavelength regions contain overlapping spectral lines that produce strong photospheric absorption of the underlying stellar continuum. To the extent that the extended diffuse gas cloud has similar abundances and temperature as the stellar photosphere, it too will produce strong absorption in these wavelength regions. Figure 11 supports this: it is 

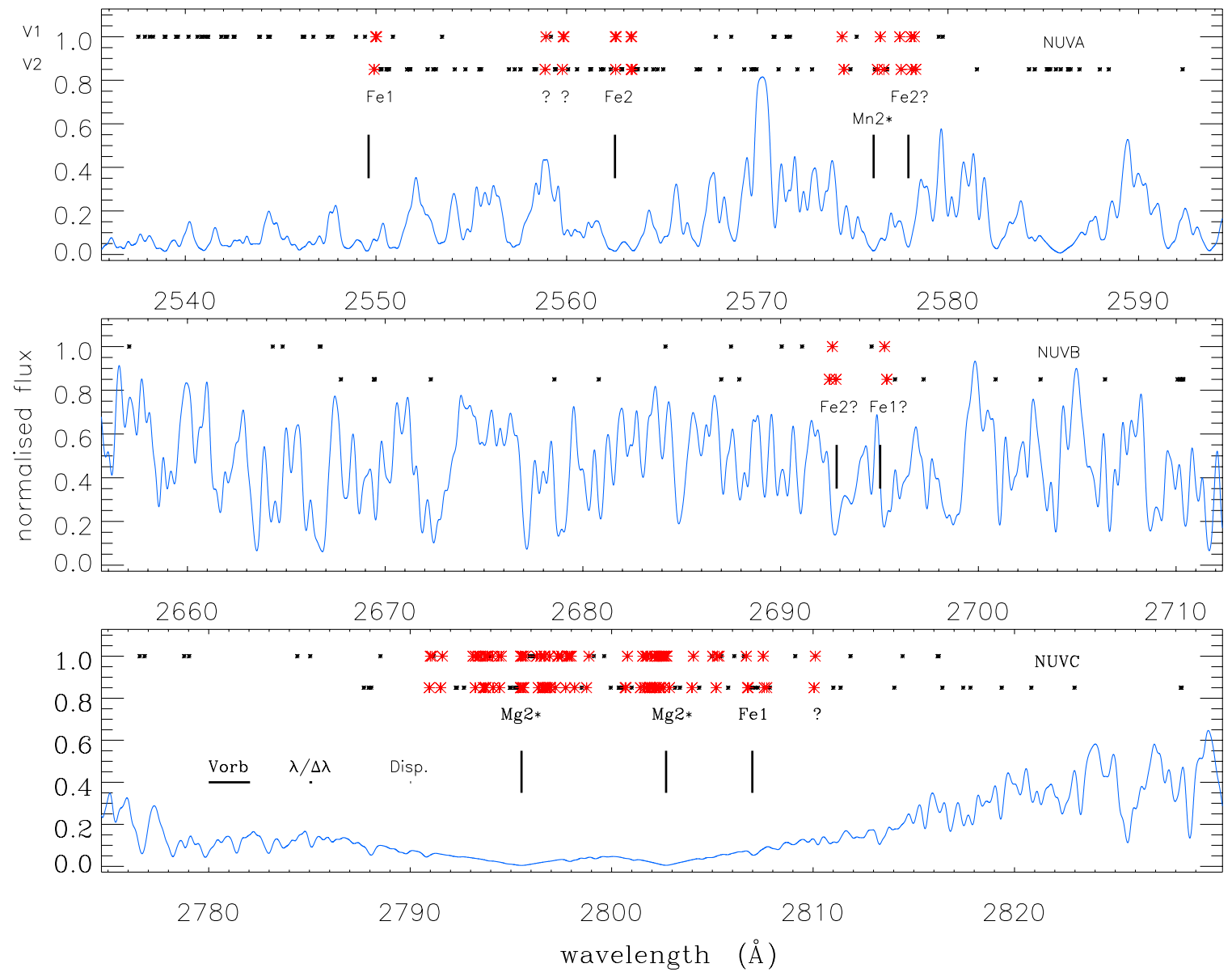

Figure 11. Wavelengths at which significantly over-deep transits were detected are indicated with a black cross, except for those wavelengths detected in both visits, where we use a large red asterisk instead. The upper row and lower rows of symbols correspond to Visit 1 and Visit 2, respectively. The horizontal scale bars in the bottom (NUVC) panel indicate the spectral dispersion, the spectral resolution, and the magnitude of WASP-12 b's orbital velocity. The blue line is the synthetic stellar spectrum, normalized to the continuum level, with a resolution of $R=10,000$, adopted to simulate the HST LSF. We do not use the synthetic stellar spectrum in our analysis. Wavelengths of a few absorbing species are identified, with a small asterisk after the ion identification for resonance lines.

(A color version of this figure is available in the online journal.)

immediately obvious that there are far more wavelengths exhibiting enhanced transit depths in NUVA and NUVC than there are in NUVB. It is also clear that the wavelengths exhibiting excess transit depths generally occur where the emergent stellar flux is low. This shows our rule of thumb works well: the photospheric absorption within a wavelength range is a good predictor of the likely diffuse absorption.

Figure 11 shows that our identified wavelengths in NUVC from the two distinct visits are largely associated with absorption in the wings of the Mg II resonance lines. This is clearly and unambiguously detected in both visits, and at so many distinct wavelength pixels the median value of the ratio spectrum must be lowered by them. Away from the cores of $\mathrm{Mg}$ II at $\sim 2788-2789 \AA, \sim 2811-2812 \AA$, and $\sim 2815 \AA$, there is a suggestion of a consistent wavelength shift between the Visit 2 (upper) and Visit 1 (lower) detections, with the Visit 1 transits being redshifted by $\sim 0.5-1 \AA\left(\sim 50-100 \mathrm{~km} \mathrm{~s}^{-1}\right)$. This shift is much greater than our spectral resolution, but less than the magnitude of the planet's orbital velocity $\left(230 \mathrm{~km} \mathrm{~s}^{-1}\right)$, which is the natural velocity scale for motions of material orbiting the star. The NUVA and NUVB identified wavelengths show less consistency between the two visits, except for wavelengths consistent with resonance lines that are picked out in both visits.
The next step is to attempt to identify the spectral lines causing these enhanced transit depths. In Paper I, we arbitrarily restricted our line identifications to resonance lines, and the consistently detected wavelengths in Figure 11 show that this was a sensible first step. Nonetheless, strong non-resonance lines of abundant elements may be more prominent than the resonance lines of rare elements. We attempted to assess this with a procedure which accounts for the stellar abundances derived for WASP-12 (Fossati et al. 2010a), the velocity shift required to match the rest wavelength of the line to the observed deviation, the excitation potential of the line, and the $g f$ value of the line. We introduce three simple functions, $P_{a}, P_{\Delta \lambda}$, and $P_{\chi}$ (each defined below), to estimate the effects of the first three of these factors on the likelihood of a particular identification being correct.

We wish to estimate the probability that an observed $3 \sigma$ deviation at wavelength $\lambda$ arises from absorption by the spectral line $i$ of element $e l$ which has rest wavelength $\lambda_{i}$. We consider a wavelength range $\lambda_{i} \pm \Delta \lambda$ and adopt $\Delta \lambda=3 \AA$ in our analysis to recover the full range of deviating points we identified in the broad wings of the Mg II lines in Paper I (see Figure 3 therein.) This corresponds to a velocity shift slightly greater than the magnitude of the orbital velocity of WASP-12 b, but our procedure favors the smallest possible value of wavelength/ velocity shift. 

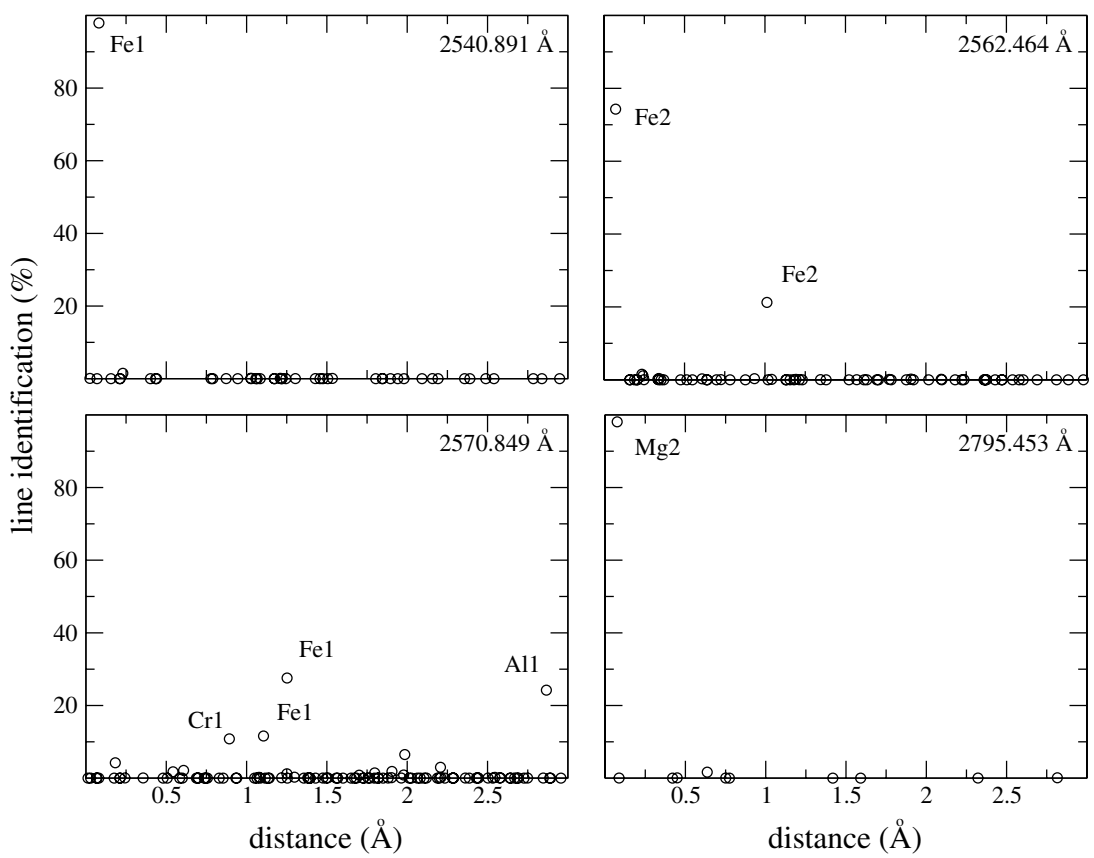

Figure 12. Line identifications from the Visit 1 data. The adopted temperature was $3000 \mathrm{~K}$ and an abundance pattern consistent with Fossati et al. (2010a) was assumed. The examples show three fairly firm identifications and one point where no firm conclusion is possible. $L_{i}$ is shown for every spectral line where it exceeded a value of $10^{-5}$, many lines considered had values of $L_{i}$ below this threshold.

The wavelength shift required to match the observed point to rest wavelength is accounted for in the factor $P_{\Delta \lambda}$; the excitation potential, $\chi_{i}$, of the lower level in the factor $P_{\chi}$; and the abundance in the factor $P_{a}$. We define these probability factors by

$$
P_{\Delta \lambda}=1-\left(\frac{\left|\lambda_{i}-\lambda\right|}{\Delta \lambda}\right) ; \quad P_{\chi}=e^{-\frac{\chi_{i}}{k T}} \quad P_{a}=\frac{N_{e l}}{Z},
$$

where $N_{e l}$ is the abundance of the element considered and $Z$ is the "metal" abundance. Each gives a value of unity for the most favorable value of the quantity concerned; $P_{a}$ is unity if the proposed gas composition comprises only $\mathrm{H}, \mathrm{He}$, and the element under consideration. $P_{\Delta \lambda}$ was designed to have a weak dependence on the shift $\left|\lambda_{i}-\lambda\right|$, as we expect the gas to have nonzero velocity w.r.t. the stellar rest frame. $P_{\chi}$ is proportional to the expected population in the lower level and $P_{a}$ is proportional to the number of absorbing atoms/ions.

$P_{\Delta \lambda}, P_{\chi}$, and $P_{a}$ are multiplied together and further multiplied by the $g f$ value of the line under consideration to give our estimated overall probability measure, $P_{i}$, that the deviation is associated with the specified spectral line, i. $P_{i}$ in Equation (3) is thus not normalized to 1 :

$$
P_{i}=\left(P_{\Delta \lambda} P_{\chi} P_{a}\right) \cdot(g f)_{i} .
$$

We then use this framework to assess the likely contribution to the measured deviation of all known spectral lines within $\Delta \lambda$. $P_{i}$ estimates the likely relative contribution of each of these lines to the detected absorption; summing over all lines estimates the total absorbing capacity, $P_{\text {tot }}$. The fraction of this due to any given spectral line is our proxy for the likelihood, $L_{i}$, that this is the correct line identification:

$$
P_{\mathrm{tot}}=\sum_{i} P_{i} ; \quad L_{i}=\frac{P_{i}}{P_{\mathrm{Tot}}}
$$

This framework indicates, with some physical justification for our estimates, how the likely line identification depends on the assumed temperature and abundance pattern. Our factor to account for a decreasing likelihood as the shift from the line's rest wavelength increases is arbitrary, but adequate, given the complex COS LSF, the unknown velocity distribution in the absorbing gas, and the $\mathrm{S} / \mathrm{N}$ of our data. The temperature dependence is only indicative: we have not used the Saha equation to determine the ionic balance. Figure 12 shows examples of applying this procedure to a selection of four points detected as having enhanced transit depths at a significance of $3 \sigma$ or more in Visit 1 . Similarly, Figure 13 shows a selection of line ID assessments from the Visit 2 data.

In Figure 12, the two upper panels reveal absorption from $\mathrm{Fe}$ I and Fe II causing enhanced transit depths at $2540.891 \AA$ and $2562.464 \AA$. The lower right panel shows the overwhelming probability that the enhanced transit depth at $2795.453 \AA$ is caused by $\mathrm{Mg}$ II. This is because the imputed line is one of the pair of resonance lines with large $g f$ values that dominate the NUVC spectral region. For this line, the $P_{\chi}$ and $g f$ factors are so large that the assumed $\mathrm{Mg}$ II abundance would need to be vanishingly small to identify any other nearby spectral line as likely to contribute significantly to the total absorption. Our Paper I detection of absorption in $\mathrm{Mg}$ II during Visit 1 remains unassailable under this more careful scrutiny. Visit 2 provides independent data, and as the lower left hand panel of Figure 13 demonstrates, it shows that the Visit 2 detection of an enhanced transit depth at $2795.307 \AA$ can be confidently attributed to $\mathrm{Mg}$ II. Note that the wavelength solutions produced by the simultaneous arc lamp spectra differ for the two visits, so we cannot compare pixels with identical wavelength sampling without rebinning. Rebinning is undesirable because adjacent pixels are then no longer independent.

Not all deviating points could be unambiguously associated with absorption by a single ionic species: the lower left panel of Figure 12 and the upper left panel of Figure 13 show examples 

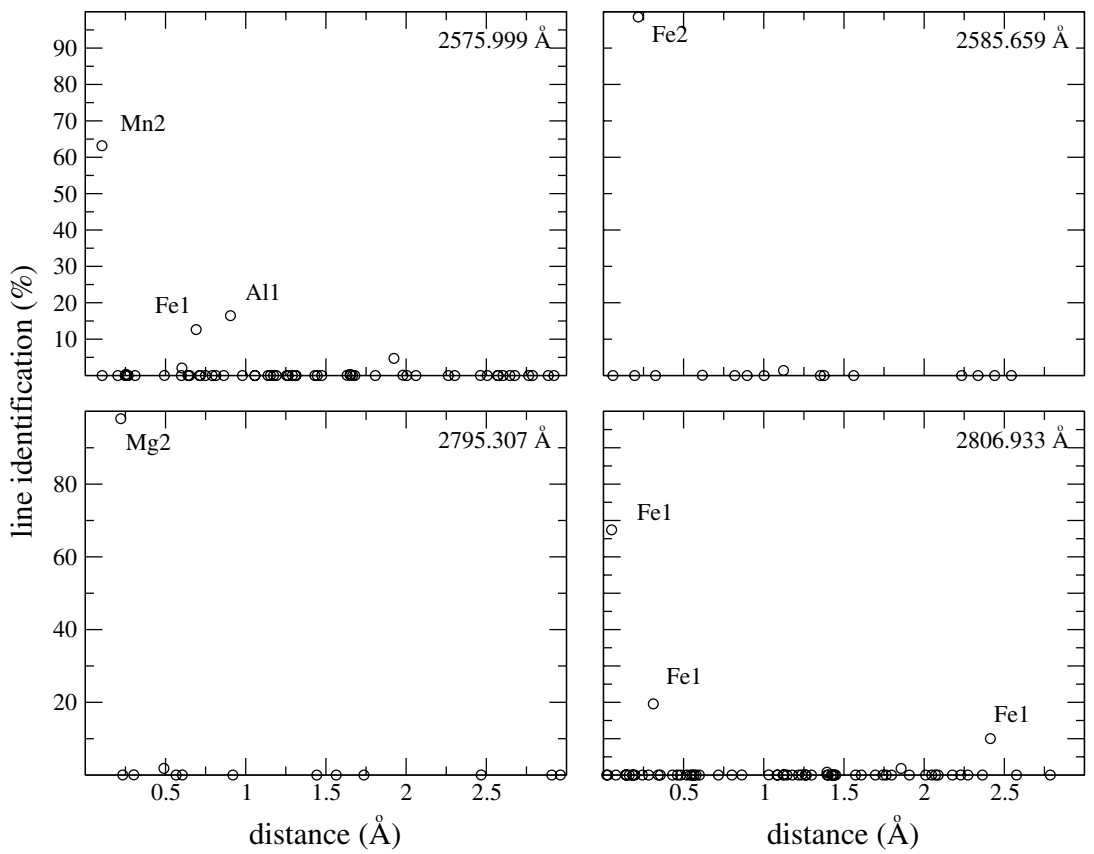

Figure 13. Line identifications from the Visit 2 data. The adopted temperature was $3000 \mathrm{~K}$, and an abundance pattern consistent with Fossati et al. (2010a) was assumed. The examples show three fairly firm identifications and one (top left) probable identification. $L_{i}$ is shown for every spectral line where it exceeded a value of $10^{-5}$, many lines considered had values of $L_{i}$ below this threshold.

where there are plausibly large contributions from a number of different elements. The detected absorption is likely to be a mix of the various spectral lines, with the dominant contributor(s) depending on the unknown composition and physical conditions of the gas. We can, however, conclude from Figures 12 and 13 that we have detected absorption from Fe I, Fe II, Mg II, and probably Mn II.

Table 5 gives the full list of wavelengths where we detected enhanced transit depths. We used a relative shift of $0.2 \AA$ as our measure of wavelength consistency; this is approximately the value of the formal resolution plus the dispersion (the wavelength solution and hence the pixelation of the ratio spectra differ between the two spectra). It is far less than the magnitude of WASP-12 b's orbital velocity, so as the motions in the gas are probably time dependent, we are imposing a very strict criterion for consistency between the two visits. Where enhanced transit depths are detected within $0.2 \AA$ of each other in both visits, these are listed in bold in Table 5. We have two independent measurements of the ratio spectrum, one from each visit, so enhanced depths which are detected at consistent wavelengths in both are unlikely to be due to noise. We would expect a match for any wavelength pixel picked out in Visit 1 to occur due to Gaussian-distributed noise in $2.7 \%$ of cases. In NUVA (NUVB, NUVC), 53\% (28\%, 70\%) of Visit 1 detections using the $\left.3 \sigma d_{i}\right|_{\text {prop }}$ statistic are matched by the same statistic in Visit 2 .

A definitive assignment of identifications to these many wavelengths exhibiting enhanced transit depths requires more certain knowledge of the properties of the diffuse gas than we currently have. We can, however, examine how changing our assumptions affects our line identifications. Figure 14 examines how the identification of the absorber at $2672.018 \AA$ depends on the assumed abundance distribution. In Paper I, by including only resonance lines in our analysis, we excluded many plausible spectral lines from our consideration. This led us to identify enhanced absorption at wavelengths around $2680 \AA$ with V II (see Table 2 of Paper I). Figure 14 shows a more careful

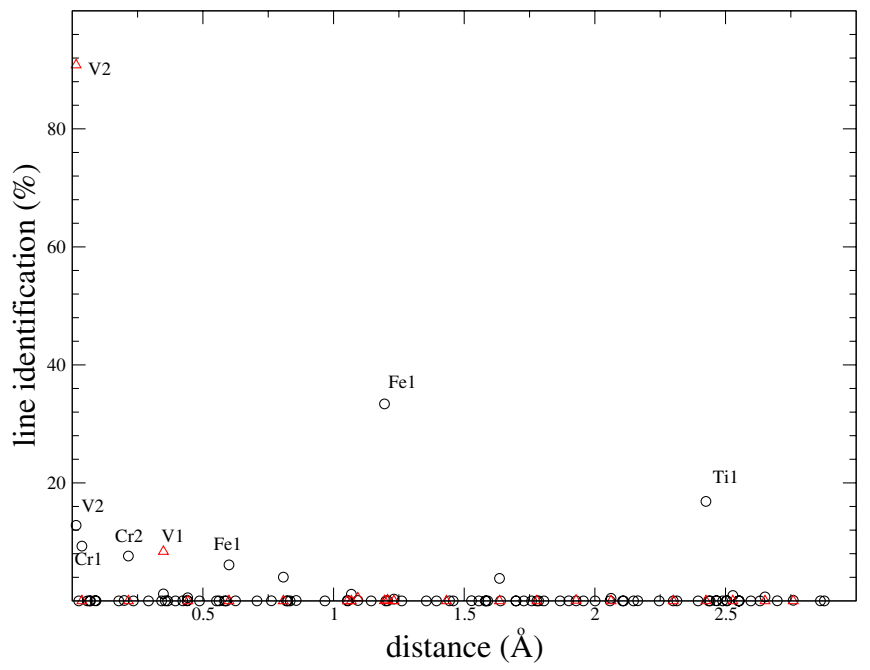

Figure 14. Results of applying our line identification procedure to the deviating point at $2672.018 \AA$. The black points assume our best estimate of the abundances of the WASP-12 stellar photosphere, while the red triangles instead apply the analysis with an arbitrary increase in the abundance of vanadium so that it has the same abundance as iron (by number). This makes it clear that the results of our line identification are strongly dependent on the assumptions we make.

(A color version of this figure is available in the online journal.)

examination of the line identification of one of these points. The black open circles show our likelihood estimates for an abundance pattern that matches our current best estimate of that of the WASP-12 stellar photosphere (Fossati et al. 2010a). With this abundance pattern, vanadium is too rare to make a dominant contribution to the absorption; instead, we are led to conclude that Fe I and Ti I are more likely than V II. Iron is generally rather abundant, and in common with many other ironpeak elements including vanadium, has a complex electronic structure, leading to a large number of blended iron lines, 


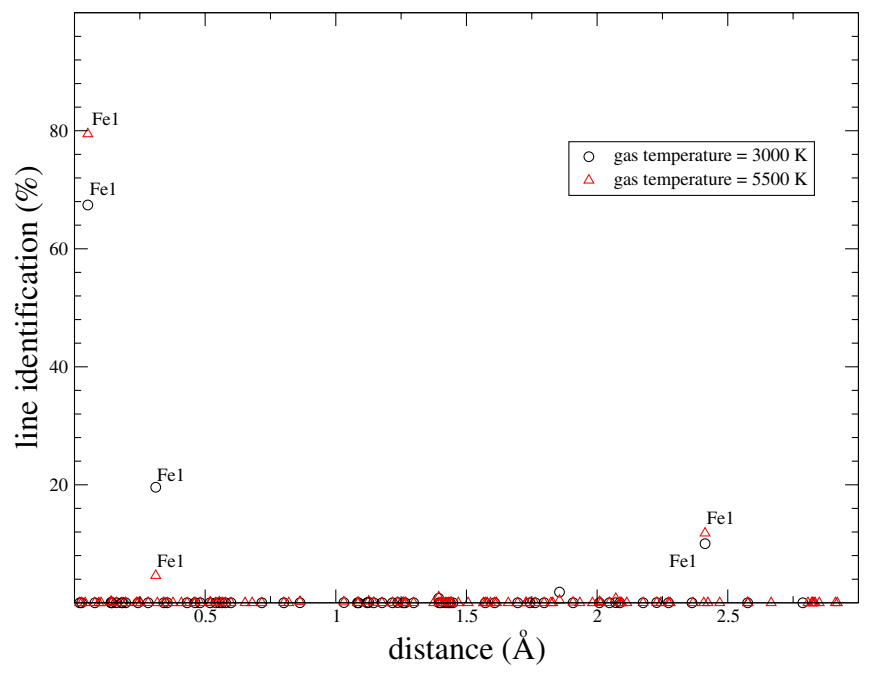

Figure 15. Results of applying our line identification procedure to the deviating point at $2806.933-T 5500 \AA$. The two sets of points correspond to $3000 \mathrm{~K}$ and $5500 \mathrm{~K}$. This makes it clear that the results of our line identifications depend on the temperature as well as the abundance pattern assumed for the absorbing gas.

particularly in the NUV. If, on the other hand, we were to assume that vanadium is abundant in WASP-12 b's exosphere and we arbitrarily increased the vanadium abundance to match that of iron in WASP-12's photosphere, we would introduce a significant change to our assessment of the line identification. The open red triangles in Figure 14 show the results we obtain if we adopt this assumption. In this case, we identify V II and V I as the species most likely to be responsible for the enhanced transit depth. It is worth noting that VO has been widely discussed as a possible constituent of the stratospheres of hot Jupiter exoplanets (e.g., Desert et al. 2008; Spiegel et al. 2010; Fortney et al. 2010), and it is possible that the gases lost from the upper atmosphere of a hot Jupiter could have enhanced vanadium abundances.

Figure 15 shows an example of how the imputed line differs for values of $3000 \mathrm{~K}$ and $5500 \mathrm{~K}$ in $P_{\chi}$, both plausible values for the exosphere of WASP-12. None of our clear identifications changed between these two temperatures, but we note that we have not included the temperature dependence of the ionization balance in our assessments.

Figures 14 and 15 show that our line identifications depend on the assumptions we make. This is generally true in astrophysics, but in more mature fields, we have a sound basis for confidently adopting likely assumptions. For example, we have a very good understanding of the physical conditions prevailing in stellar atmospheres, and much high $\mathrm{S} / \mathrm{N}$ data have been used to hone models such as the one we plotted in Figures 1 and 11. The study of hot Jupiter exospheres is not so well developed.

To be sure of all line identifications in WASP-12 b and other hot Jupiters, we need sound assumptions for the likely abundances in the absorbing material, and measurements of its physical properties. Hot Jupiter atmospheres probably have prodigious winds and disequilibrium chemistry which will affect the abundances of the exospheric material (e.g., Moses et al. 2011). The models have many degrees of freedom, and for WASP-12 b constraining them will be challenging, as the system is relatively distant (380 \pm 85 pc; Fossati et al. 2010a) and hence at the limit of what we can do with HST/COS. WASP$12 \mathrm{~b}$ is roughly the most distant exoplanetary system for which

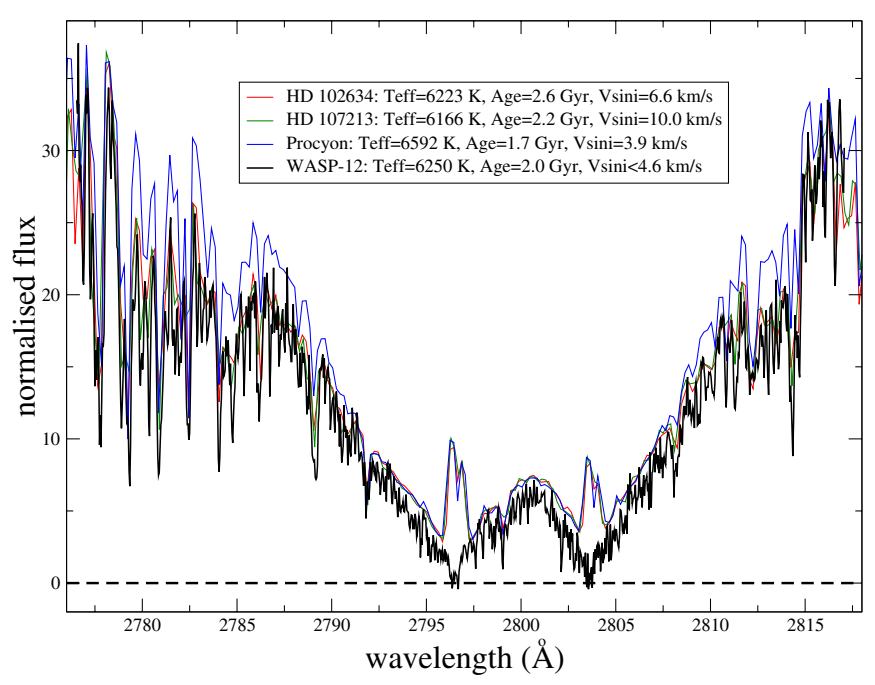

Figure 16. Our Visit 1 NUVC coverage of the cores of the Mg II resonance lines compared with those of three other stars (see the text). The cores of the WASP12 lines show no emission whatsoever and are suggestive of narrow absorption components that reduce the flux in the cores of these lines to zero. WASP-12 is remarkable in the appearance of its $\mathrm{Mg}$ II resonance line cores.

NUV transit observations can realistically be obtained with technology available in the foreseeable future. The best chance to put our understanding of hot Jupiter exospheres on a firm foundation would be to observe the two brightest hot Jupiters, HD 209458 b and HD 189733 b in the NUV. In some cases, however, we already have robust identifications of spectral lines exhibiting enhanced transit depths in WASP-12 b, for example for the $\mathrm{Mg}$ II resonance lines.

\subsection{Chromospheric Activity in WASP-12 and Tenuous Gas Surrounding the System}

Our HST/COS data raise questions concerning stellar activity in WASP-12. Can we attribute the high point at phase $\phi=0.95$ in the Visit 2 light curves to stellar activity as we speculated in Section 3.3? Fossati et al. (2010a) found an age of 1.0-2.65 Gyr for WASP-12, and an effective temperature of $T_{\text {eff }}=6250 \pm$ $100 \mathrm{~K}$, in agreement with Hebb et al. (2009) but with tighter uncertainties. Generally, stars of this $T_{\text {eff }}$ and age are expected to exhibit activity, and emission cores in the $\mathrm{Mg}$ II resonance lines are one clear observable consequence of this. As Figure 16 demonstrates, however, $\mathrm{Mg}$ II emission cores are conspicuously absent in WASP-12.

To produce Figure 16, we normalized the spectra of WASP-12 and two stars of the same age and $T_{\text {eff }}$ to match the flux in the far wings of the $\mathrm{Mg}$ II lines (the regions used in the normalization are further from the line cores than the edges of Figure 16). These two stars, HD 102634 and HD 107213, are plotted in red and green in Figure 16. As expected for such similar stars, the line profiles of WASP-12, HD 102634, and HD 107213 match well throughout the profile of the $\mathrm{Mg}$ II lines, except within about $10 \AA$ of either of the line cores, where WASP-12's spectrum lies below the others. Figure 16 also shows Procyon, which is a commonly used reference star, slightly hotter, slightly younger, and more slowly rotating ( $v \sin i=3.9 \mathrm{~km} \mathrm{~s}^{-1}$; Schroder et al. 2009) than the other three objects. Procyon is noted for its low stellar activity. Procyon, HD 102634, and HD 107213 all have very similar line profiles between $2792 \AA$ and $2807 \AA$, with similar emission cores in all three cases surrounded by fairly steep declines in flux toward the line cores, with very similar 
gradients in all three examples. WASP-12 has anomalously low fluxes within about $10 \AA$ of the $\mathrm{Mg}$ II line cores, with steeper gradients toward the line cores and absolutely no sign of emission in the line cores.

Figure 16 shows that WASP-12, HD 102634, and HD 107312, which have similar ages and temperatures, match extremely well, except for in the line cores. Indeed, the WASP-12 line cores show no sign of the anticipated emission reversals and appear essentially as saturated absorption profiles: the line cores have zero flux. As the point-to-point deviations in the WASP12 spectrum make clear, we would definitely have detected emission cores in WASP-12 even if they were present at a strength significantly less than that in the three comparison stars. Even if WASP-12 were a slowly rotating sub-giant, Mg II emission cores would be expected (Ayres 2010). Even very inactive Sun-like stars have significant chromospheric spectra with prominent $\mathrm{Mg}$ II emission powered by acoustic shock heating and local dynamo action that converts hydrodynamic turbulence into magnetic flux.

Our interpretation of Figure 16 is that the inner parts of WASP-12's Mg II lines are absorbed by gas beyond the stellar chromosphere, with sufficient column density to completely obliterate the expected emission reversals, and to perhaps also depress the line wings just outside the cores. Because the star is distant, one possibility is interstellar absorption. As we demonstrate below, however, the necessary $\mathrm{Mg}^{+}$column density is quite substantial and is implausible unless the interstellar medium (ISM) in that direction is unusual. Another possibility, which we favor, is that the absorption is local to the WASP-12 system.

Gas immediately surrounding WASP-12 b cannot account for the absorption we have just described. The planet itself covers only a small fraction of the stellar disk: to completely remove the predicted $\mathrm{Mg}$ II chromospheric emission would require a much more spatially extended distribution of absorbing material, blanketing the entire stellar disk. We propose, in fact, that the entire system is shrouded in diffuse gas, very likely stripped from WASP-12 b itself under the harsh radiation and stellar wind conditions so close to the parent star. Knutson et al. (2010) measured the $\mathrm{Ca}$ II $\mathrm{H}$ and $\mathrm{K}$ emission lines of 50 transiting planet host stars including WASP-12. WASP-12's Ca II H \& K lines are completely devoid of detected emission cores, as are the majority of Knutson et al.'s (2010) sample. Knutson et al. (2010) interpreted their results in terms of a correlation between stellar activity and hot Jupiter atmosphere type, but our hypothesis suggests an alternative/additional explanation. Many of these systems could be shrouded in diffuse gas, which absorbs any emission cores produced by stellar activity.

Chromospheric activity is strongly correlated with stellar age and rotational velocity. WASP-12's rotational velocity is unknown, but the transit of WASP-12 b is accompanied by an undetected Rossiter-McLaughlin effect (Husnoo et al. 2011), so either the rotational velocity is low or the orbital angular momentum of WASP-12 b is misaligned with the stellar rotation axis (see, e.g., Haswell 2010, for explanation). If the chromospheric activity is low, as expected for a middle-aged slowly rotating dwarf, then a stellar flare is a very unlikely explanation for the high flux in orbit 3 of our Visit 2 light curves. We must then conclude that either our data are intrinsically noisy beyond the assigned photometric error or that we are viewing WASP-12 through diffuse gas at all observed orbital phases, and there happened to be a relatively clear view to the stellar surface during orbit 3 of Visit 2. This latter possibility might be produced

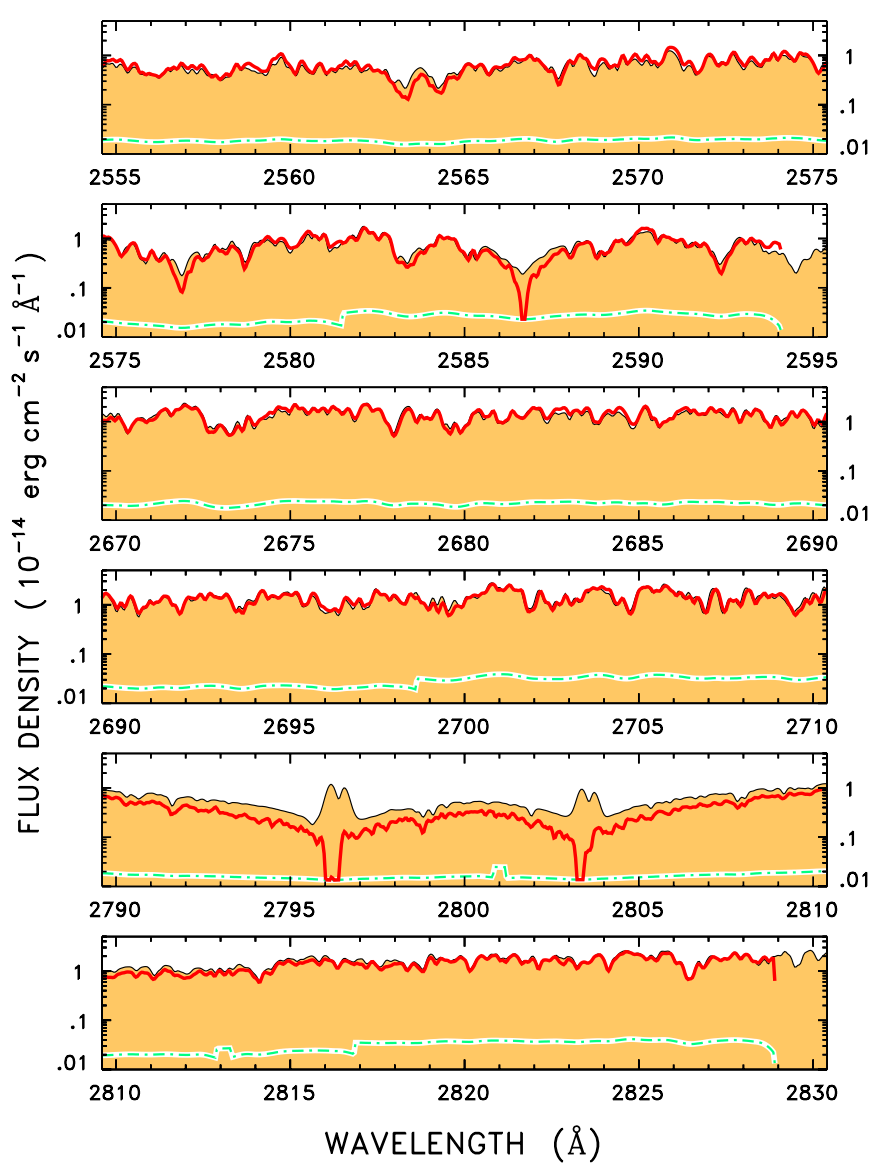

Figure 17. Our WASP-12 NUV spectrum (red) in comparison to that of $\alpha$ Cen (black line, filled beneath). A logarithmic flux scale has been adopted to emphasize the deepest absorption. The green dotted line indicates the errors on the WASP-12 spectrum: data are truncated at levels consistent with zero flux.

by a bow shock in a very extended planetary magnetosphere (Vidotto et al. 2011).

\subsection{Resonance Line Absorption in WASP-12 and the ISM}

Figure 17 shows our Visit 2 WASP-12 spectrum compared to HST/STIS data on $\alpha$ Cen from the archive. The Space Telescope Imaging Spectrograph (STIS) data have been convolved with the COS NUV LSF and scaled by the multiplicative factor $4 \times 10^{-5}$. Both spectra have been smoothed for display purposes. The flux axis is logarithmic to emphasize the deep absorption in the cores of strong lines. The photometric error is per resolution element, and the flux densities have been truncated at the $1 \sigma$ error level. As in Figure 16, the spectra have been normalized to agree in the far wings of the Mg II absorption. Generally, stellar Mg II line profiles scale so that normalizing in the far wings produces good agreement in the inner wings for stars of the same luminosity class (Ayres 2010), but as we saw in Figure 16, WASP-12's inner wings are depressed relative to other main sequence stars. The $\mathrm{Mg}$ II resonance line cores in WASP-12 have total absorption, which is perhaps blueshifted by $\sim 20 \mathrm{~km} \mathrm{~s}^{-1}$.

In the NUVA region of Figure 17, WASP-12 exhibits similar total absorption in the core of the Fe II resonance line at $2586 \AA$, where $\alpha$ Cen's absorption is shallower by over a factor of 10 . Appearing slightly less strong is WASP-12's Mn II resonance line at $2577 \AA$.

These absorption features in the WASP-12 stellar spectrum are too deep to have their origin in the photosphere itself. Our 


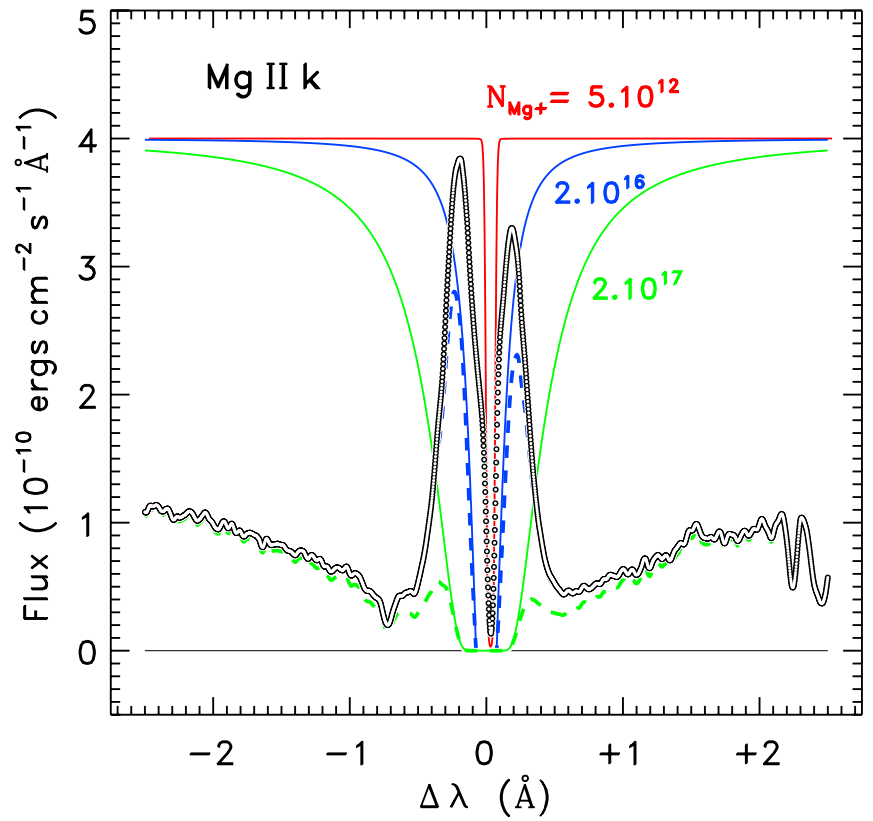

Figure 18. Calculations of interstellar absorption applied to the center of the $\mathrm{Mg}$ II line profile of $\alpha$ Cen. The observed line profile is plotted as open circles that overlap to form a double line; the solid lines show the predicted absorption for column densities of $N\left(\mathrm{Mg}^{+}\right)$of $5 \times 10^{12} \mathrm{~cm}^{-2}$ (red), $2 \times 10^{16} \mathrm{~cm}^{-2}$ (blue), and $2 \times 10^{17} \mathrm{~cm}^{-2}$ (green). The modified line profiles produced by applying the two higher column densities are indicated in dashed lines of corresponding color.

favored interpretation is that the absorption arises in gas within the WASP-12 system, but we now examine the plausibility of the alternative explanation that the absorption might arise in the ISM.

Figure 18 shows the central part of the observed $\alpha$ Cen A $\mathrm{Mg}$ II k-line profile. The solid red line shows the absorption we expect from the ISM along the very short pathlength $(1.3 \mathrm{pc})$ to the star, using the Mg II column density of $5 \times 10^{12} \mathrm{~cm}^{-2}$ reported by Linsky \& Wood (1996). The absorption profile was calculated using a temperature of $7000 \mathrm{~K}$ and a turbulent velocity of $1.5 \mathrm{~km} \mathrm{~s}^{-1}$, typical values for the local warm cloud in which $\alpha$ Cen A and the Sun are embedded. The calculated absorption fits the narrow slightly redshifted absorption dip in the extreme core of the line, noting that the broader "absorption" between the twin peaks of the profile is a central reversal of chromospheric origin (see Linsky \& Wood 1996). The blue curve indicates the attenuation predicted for a plausible $N\left(\mathrm{Mg}^{+}\right)$ in the line of sight (LOS) to WASP-12: $2 \times 10^{16} \mathrm{~cm}^{-2}$. We arrived at this estimate assuming $E(B-V) \sim 0.2 \mathrm{mag}$ for WASP-12 (based on a nominal 0.6 mag kpc $\mathrm{kp}^{-1}$ of reddening for an average Galactic sight line and the $\sim 350 \mathrm{pc}$ estimated distance of WASP-12), which yields a hydrogen column density of $\sim 1.2 \times 10^{21}$ from the standard Milky Way reddening law (e.g., Savage \& Mathis 1979). If we then assume that roughly half the magnesium is in the form of $\mathrm{Mg}^{+}$- about what is deduced from the $\alpha$ Cen A sight line, albeit perhaps not typical-then we obtain: $N\left(\mathrm{Mg}^{+}\right) \sim 2 \times 10^{16} \mathrm{~cm}^{-2}$. Applying the corresponding absorption profile to the $\alpha$ Cen $\mathrm{A} \mathrm{MgII} \mathrm{k}$ line results in the dashed blue curve, which still preserves substantial core emission.

The green line in Figure 16 depicts the absorption profile for an $\mathrm{Mg}$ II column density a factor of 10 greater than the interstellar estimate above, i.e., $N\left(\mathrm{Mg}^{+}\right)=2 \times 10^{17} \mathrm{~cm}^{-2}$. Here, broad damping wings have developed, strongly suppressing the

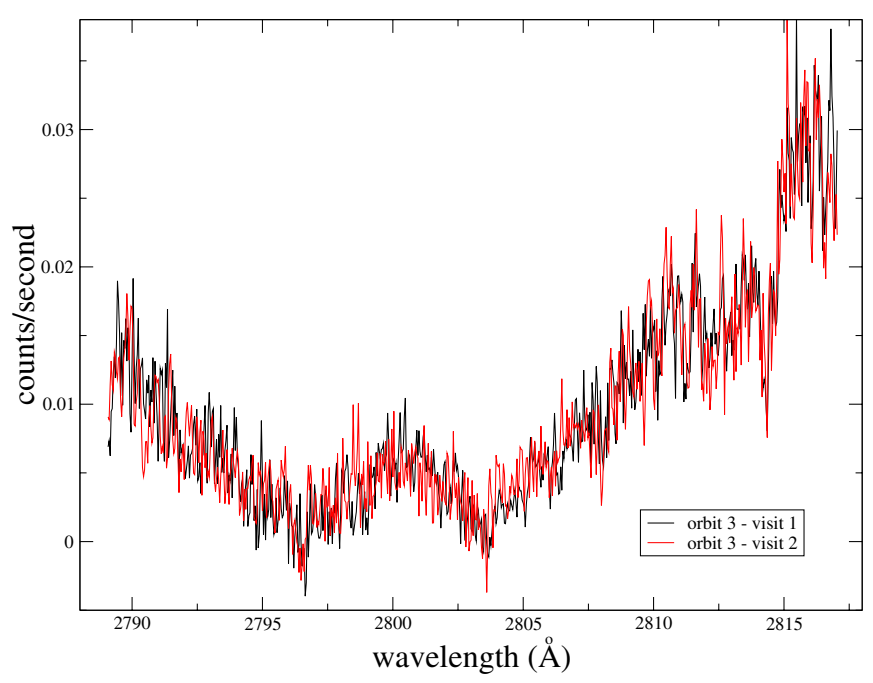

Figure 19. Direct comparison of the NUVC overlapping spectral region in orbit 3 of each of the two visits.

emission core, and even affecting the inner wings of the $\mathrm{k}$ line. While the predicted profile is not an exact match to that of WASP-12 (cf. Figure 14), it does demonstrate the plausibility of the absorption mechanism, albeit requiring a very substantial column of $\mathrm{Mg}^{+}$. The required column is excessive enough, in fact, to make an interstellar origin seem much less plausible than a more local source, especially given the existence of the hot Jupiter and the possibility of strong atmospheric stripping. We examine this possibility in more detail later (Section 4.2 below).

\section{DISCUSSION AND CONCLUSIONS}

The conclusions we reached in Paper I more or less stand in the light of our Visit 2 data and our interpretation of the entire COS/NUV data set. As Section 3 shows, the Visit 2 data did not neatly fill in the phase-folded NUV light curves we produced from Visit 1. Instead, a rather more complex reinterpretation was required. Our principle conclusions in Paper I (from Visit 1 data alone) were that the NUV transit depth is deeper than the optical, that this is due to absorption in metal atoms/ions within WASP-12 b's exosphere, and that this exospheric gas was spatially distributed such that an early ingress occurs. To make sense of our combined NUV light curves, we needed to adopt two further hypotheses: (1) the absorbing gas is (at least sometimes) even more spatially extended than we inferred from Visit 1 and (2) a short-lived stellar flare occurred during Visit 2 orbit 3 or Visit 2 orbit 3 had an LOS through a relatively low density window in the absorbing gas.

In Section 3, we presented several lines of indirect evidence in favor of the hypothesis that WASP-12 has some chromospheric activity, despite the resounding lack of observed emission cores in the $\mathrm{Mg}$ II and $\mathrm{Ca}$ II lines. We now examine our data to see if it contains direct evidence in favor of this hypothesis over the alternative low density window hypothesis.

\subsection{A Stellar Flare During Visit 2 Orbit 3 ?}

We have attributed the high NUV fluxes during Visit 2 orbit 3 to a stellar flare. To check this interpretation, Figure 19 shows a direct comparison of the NUV spectra from orbit 3 of Visit 2 with orbit 3 of Visit 1. The two spectra are plotted with the pipeline calibration, no normalization was applied to account for the declining throughput. These spectra are clearly noisier 
that those shown in earlier figures, as they comprise only about a fifth of the exposure time on each visit. The two spectra broadly agree, but there is a hint in the Visit 2 spectrum of a small emission component redward of the center of each of the Mg II line cores. This is not an unassailable detection. The appearance of these two small bumps is very similar to the remnant line core emission on the red side of the line profile in Figure 18 after application of absorption by a column of $N\left(\mathrm{Mg}^{+}\right)=2 \times 10^{17} \mathrm{~cm}^{-2}$. The remnant emission on the blue side of the line core is absent in Figure 19, but as we noted in our description of Figure 17, the absorption in WASP-12's line cores appears slightly blueshifted (by about $0.25 \AA$ ) relative to the photospheric spectral lines. The damping wings of a blueshifted absorption profile (see Figure 18) would extinguish the blue side of the emission core before the red side.

Thus, Figure 19 provides us with (noisy) direct evidence for chromospheric emission in the Mg II line cores. Furthermore, it shows an increase in this emission or a decrease in the absorption between the stellar chromosphere and the telescope during Visit 2 orbit 3.

The dramatic movement of the Visit 2 orbit 3 NUVA data point when Figures 5 and 9 (or Figures 7 and 8) are compared suggests that we are seeing a veiled stellar flare rather than a low density window. In Figures 5 and 8, the Fe II resonance line at $2586 \AA$ is included and the flux is high. In Figures 7 and 9, this line is excluded and the flux is much lower. A flare would increase the chromospheric emission in the $\mathrm{Mg}$ II and Fe II resonance lines. When the NUVA data exclude the Fe II line (Figure 9), there is no sign of a high point in the photometry. It is only where chromospheric activity indicators are included within the passband, i.e., always in NUVC (Figures 5, 7, 8, and 9) and in NUVA in Figures 5 and 8 but not Figures 7 and 9, that the light curve has a high point. We infer that the enhanced chromospheric emission in Fe II is absorbed by the obscuring gas, and re-emitted isotropically and across the line profile. Thus, the line core emission is distributed across the line profile and rendered undetectable at any individual pixel in the spectrum. When we sum over wavelength to produce light curves, however, the signal becomes apparent above the noise.

Since the flux from the stellar photosphere in the Fe II $\lambda 2586$ line is low (Figure 1), whether or not this line is included within the wavelength coverage should make little difference to the light curve in the low density window hypothesis. We therefore interpret the $4 \sigma$ movement of the Visit 2 orbit 3 NUVA point between Figures 7 and 8, i.e., between data including and excluding this line, as evidence in favor of the stellar flare hypothesis over the low density window hypothesis. Only when we include features which we expect to show a significant flux increase during a stellar flare do we see the NUV flux in Visit 2 orbit 3 lying significantly above the other data. Several aspects of our data thus suggest that we caught a stellar flare which occurred during the ingress of the optical transit in Visit 2.

\subsection{The Column Density of $M g$ II}

In Section 3.6, we showed that a high column density of Mg II between us and the stellar chromosphere can absorb WASP-12's $\mathrm{Mg}$ II emission cores, assuming that the intrinsic emission is similar to that of $\alpha$ Cen. Here, we consider whether the value we deduced, $N\left(\mathrm{Mg}^{+}\right) \sim 2 \times 10^{17} \mathrm{~cm}^{-2}$, can be plausibly attributed to mass loss from the planet WASP-12 b. Since this extremely close-in planet is the most obviously unusual characteristic of the star, it seems likely that the anomalous Mg II line profiles are ultimately caused by the planet.

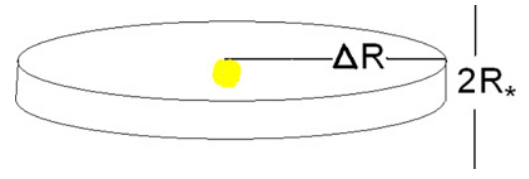

Figure 20. Geometry assumed in our estimate of plausible limits to the column density due to mass loss from WASP-12 b. Material is assumed to be spread within a squat cylinder of radius $\Delta R$ and height $2 R_{*}$ centered on the star.

(A color version of this figure is available in the online journal.)

Various mechanisms have been proposed for mass loss from WASP-12 b and similar planets. There are several variants of the "blow-off" hypothesis, which assumes hydrodynamic outflow driven by energy input from irradiation by the host star. Tidal heating can drive planetary envelope expansion and consequent Roche lobe overflow (Li et al. 2010). For the early eccentricity estimates of WASP-12 b, for example, this mechanism was

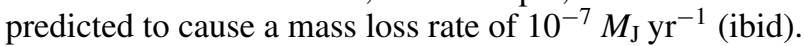

Mass lost from the planet will carry specific angular momentum from the planet's orbit and will thus tend to be confined to the orbital plane. To absorb chromospheric emission from the entire visible disk of the star, the material must extend about one stellar radius above and below the orbital plane. To derive an upper limit on the absorbing column density due to mass lost from the planet, therefore, we begin with the geometry shown in Figure 20. Gas is present within a squat cylinder of radius $\Delta R$ and height $2 R_{*}$, centered on the star and aligned with the orbital plane.

The mean density, $\bar{\rho}$, within the cylinder is

$$
\bar{\rho} \leqslant \frac{\dot{m} \Delta t}{2 \pi \Delta R^{2} R_{*}},
$$

where $\dot{m}$ is the mass loss rate and $\Delta t$ is the time over which mass has been lost. The equality arises when all mass lost from the planet is confined to the cylinder we require to be populated. This is obviously an upper limit as some mass will accrete on to the star or diffuse in the vertical direction out of our line of sight (LOS) to the star.

The column density of particles is

$$
N_{\text {tot }} \leqslant \int_{\text {LOS }} \frac{\rho}{\mu} \mathrm{d} l \approx \frac{\dot{m} \Delta t}{2 \pi \Delta R R_{*} \mu} \approx \frac{\dot{m}}{2 \pi v R_{*} \mu},
$$

where the integral is carried out along our LOS, $\mu$ is the mean molecular weight of the gas, and $v=\Delta R / \Delta t$ is the velocity with which the gas moves outward from the orbit of the planet. We have used the mean density and implicitly assumed that the velocity is approximately constant for outflow over the distance $\Delta R$. The integral is effectively performed over the distance $\Delta R$ which contributes appreciably to the column density. This avoids addressing our ignorance of the functions $\rho(l)$ and $v(l)$. The approximations are sufficient for an order of magnitude estimate, particularly since our empirical estimate of $v$ will ensure that our approximation is weighted to the appropriate part of the velocity field. If the fraction of the total number density in the form of $\mathrm{Mg}$ II is $f$, then

$$
N\left(\mathrm{Mg}^{+}\right) \leqslant \frac{\dot{m} f}{2 \pi v R_{*} \mu} .
$$

To obtain $N\left(\mathrm{Mg}^{+}\right)$, we adopt the mass loss rate predicted by evaporation processes, for example, Ehrenreich \& Desert (2011) give $\dot{m} \sim 3 \times 10^{7} \mathrm{~kg} \mathrm{~s}^{-1}$ for WASP-12 b. We take $f \approx 10^{-5}$, 
which is based on a number fraction of $10^{-4}$ for $\mathrm{Mg}$ in the solar elemental abundance mix, and a (conservative) assumption that 1 in 10 magnesium nuclei are in the $\mathrm{Mg}$ II ionic state; $R_{*}=$ $1.1 \times 10^{9} \mathrm{~m}$ (Chan et al. 2011); we assume a mean molecular weight of $\mu=m_{\mathrm{p}}=1.7 \times 10^{-27} \mathrm{~kg}$ which will be correct to within a factor of a few for material predominately composed of hydrogen. We can estimate $v$ from our data: the absorption appears to be blueshifted by roughly $0.25 \AA$ (Figure 17, fifth panel from the top), implying $v \sim 2.5 \times 10^{4} \mathrm{~m} \mathrm{~s}^{-1}$. Using these values in Equation (7), we obtain $N\left(\mathrm{Mg}^{+}\right) \leqslant 4 \times 10^{21} \mathrm{~cm}^{-2}$. The required column density is below this crude upper limit by a factor of $\sim 2 \times 10^{4}$. Obviously, some material will move inward and be captured by the star, and some will spread in the vertical direction beyond the cylinder we require to be filled with gas, but we have a comfortable factor to allow for this and other losses of $\mathrm{Mg}$ II.

\subsection{Exospheric Gas or Coronal Gas?}

WASP-12 b is only about one stellar diameter from the stellar surface, so this planet is orbiting within the star's corona. This raises the possibility that absorption we observed around WASP$12 \mathrm{~b}$ may be attributable to entrained or bow shocked coronal gas, rather than from mass lost from the planet (e.g., Vidotto et al. 2010, 2011). Indeed, Vidotto et al. (2011) show how a "double" transit can arise if a transiting planet is preceded by a bow shock in a very extended planetary magnetosphere. This does not completely explain our Visit 2 light curves, as the observed absorption begins very early and the curves which do not contain activity-indicating lines differ in shape from those which do (see Section 4.1). The typical coronal temperatures at the distance of the planet are, however, probably inconsistent with the absorption we detect. Figure 11 gives us very strong indications that the absorption causing our NUV transit is from gas at a similar temperature to the stellar photosphere. The densities in the gas causing the NUV transit exceed those of a typical stellar corona (otherwise, the NUV transit would be indistinguishable from the optical transit), so naturally, by energy arguments, the temperature of this gas is lower. This argument does not, however, rule out entrainment of coronal gas by the planet. Section 4.2 strongly suggests, however, that most of the gas around WASP-12 is due to mass loss from the planet: stars without extreme planets like WASP-12 b offer us clear sight of their Mg II chromospheric emission cores. Our observations show that the emission cores are completely absorbed throughout our temporal coverage. This requires gas at considerable distances from the planet itself.

\subsection{The Roche Lobe of WASP-12 b}

Figure 21 shows the Roche lobe of WASP-12 b. The $y-z$ crosssection is approximately that presented to us during transit; $i=86^{\circ} \pm 3^{\circ}$ (Chan et al. 2011), so the $y-z$ plane is inclined to the plane of the sky by $4^{\circ} \pm 3^{\circ}$. The lowest panel of Figure 21 shows that the cross-section of the critical equipotential surface is not much larger than that of the planet itself. We have detected transit depths in the NUVA and NUVC spectra regions which are up to a factor of three deeper than the optical transit (see Figures 5, 9 and 10). As the optical depth of the absorbing gas will vary with both wavelength and position, it is clear that we require the projection of the spatial distribution of the absorbing gas on the plane of the sky to exceed that of the Roche lobe by a factor of more than a few. Our observations thus show that the absorbing gas far overfills the planet's Roche lobe. This is
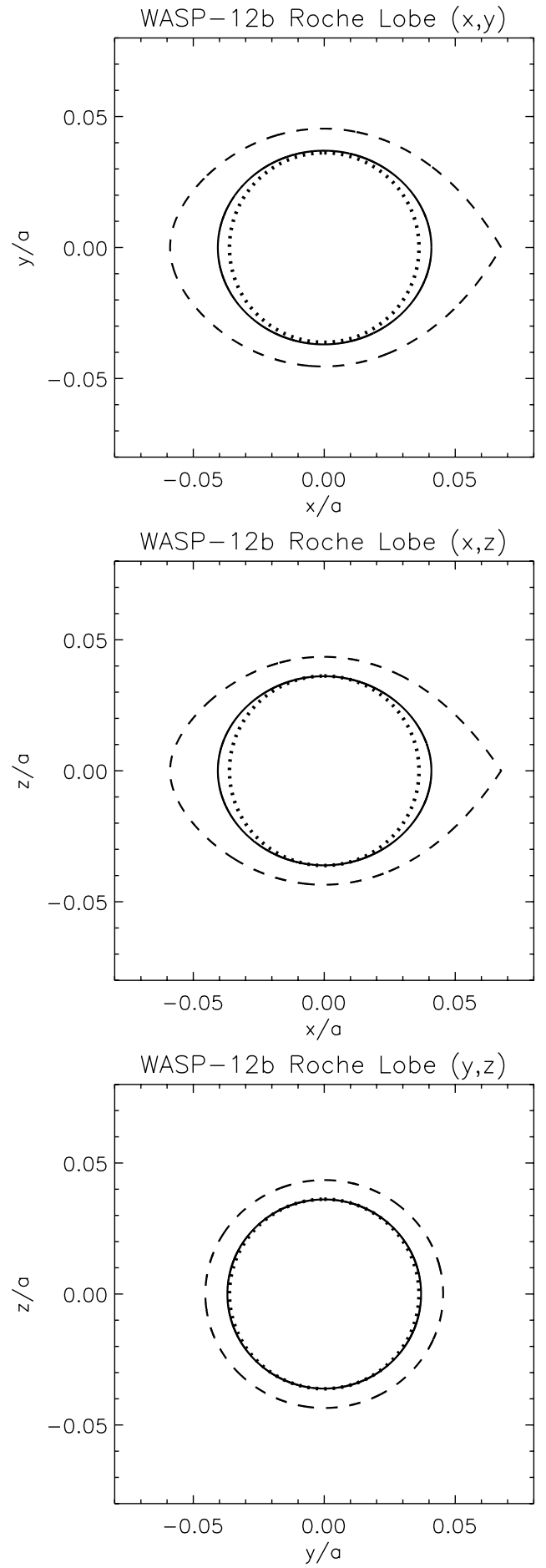

Figure 21. Roche lobe of WASP-12b. The $y-z$ cross-section (lowest panel) is presented to us during transit. The solid black lines indicate the equipotential surface defined by the area determined by the optical transit. A fluid planet will fill this surface. The dotted circles have area equal to the $y-z$ cross-section of the planet. The dashed lines show the critical equipotential surface which delimits the planet's Roche lobe.

consistent with our Visit 2 data, which suggest that absorption is occurring at phase 0.83 (see Figure 8), i.e., the absorbing gas extends $\sim 20 \%$ of the way around the orbit.

Figure 21 also shows the deviation from spherical of WASP$12 \mathrm{~b}$. The fluid planet will fill the equipotential surface corresponding to the area occulted in the optical transit. The $y-z$ cross-section of this equipotential surface deviates slightly from 


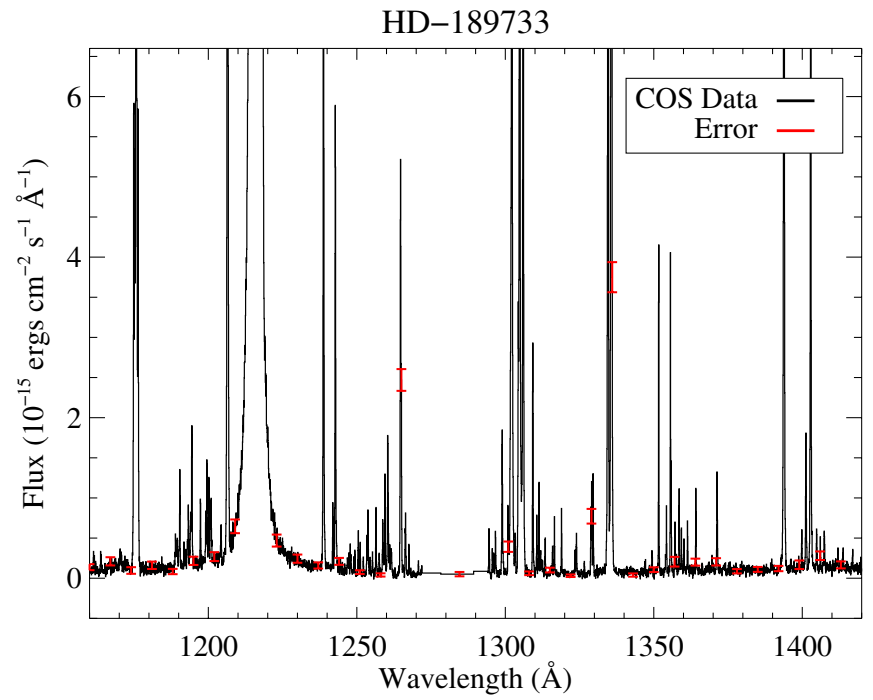

Figure 22. Co-added far-UV observations of the well-studied exoplanet host HD 189733, obtained with the HST-Cosmic Origins Spectrograph. Despite the relatively low flux levels, $\mathrm{COS}$ can produce high $\mathrm{S} / \mathrm{N}$ emission line spectra of planet host stars.

(A color version of this figure is available in the online journal.)

circular as a result of the centripetal force, with the $x-y$ and $x-z$ cross-sections each being noticeably distorted by the combination of the tidal distortion due to the stellar gravity and the centripetal force. The volume of the planet is consequently $\sim 20 \%$ greater than that of a sphere producing the same optical transit depth.

\subsection{NUV versus FUV}

As we discussed in the introduction, most of the observations of diffuse gas around hot Jupiter exoplanets have been carried out in the FUV, with our WASP-12 program being the single published exception. Our observations are clearly limited by the $\mathrm{S} / \mathrm{N}$ of the data, but it must be remembered that WASP12 is 4 mag fainter than either HD 209458 or HD 189733, the two objects where FUV transit observations exist. Even with the 4 mag advantage, conclusions from the FUV transit data can be compromised by the fact that the FUV stellar emission is patchy and variable in time and/or severely limited by poor $\mathrm{S} / \mathrm{N}$. To demonstrate how FUV observations can be irrevocably compromised by these issues, we present a brief case study using FUV COS data. The target, HD 189733 b, is a hot Jupiter transiting a bright nearby early K-star, and has been widely and informatively studied.

The observations were obtained on 2009 September 16 as part of HST program 11673. The data were acquired with the FUV G130M mode (Osterman et al. 2011), spanning $1134 \leqslant \lambda \leqslant$ $1429 \AA$ at a velocity resolution of $\approx 17 \mathrm{~km} \mathrm{~s}^{-1}$. A total of $9.9 \mathrm{ks}$ of exposure time was obtained over five spacecraft orbits, allowing time for instrumental settling and stabilization (see VidalMadjar et al. 2004 and references therein) prior to the transit observations. Observations covered orbital phases from -0.074 to 0.051 (adopting the orbital parameters of Knutson et al. 2007). The data were obtained from the Multi-Mission Archive at STScI (MAST) and calibrated one-dimensional spectra were coadded with the custom IDL procedures described by Danforth et al. (2010). The co-added spectrum is displayed in Figure 22. The $\mathrm{S} / \mathrm{N}$ per resolution element is $>50$ in the chromospheric C II $\lambda \lambda 1334,1335$ lines.

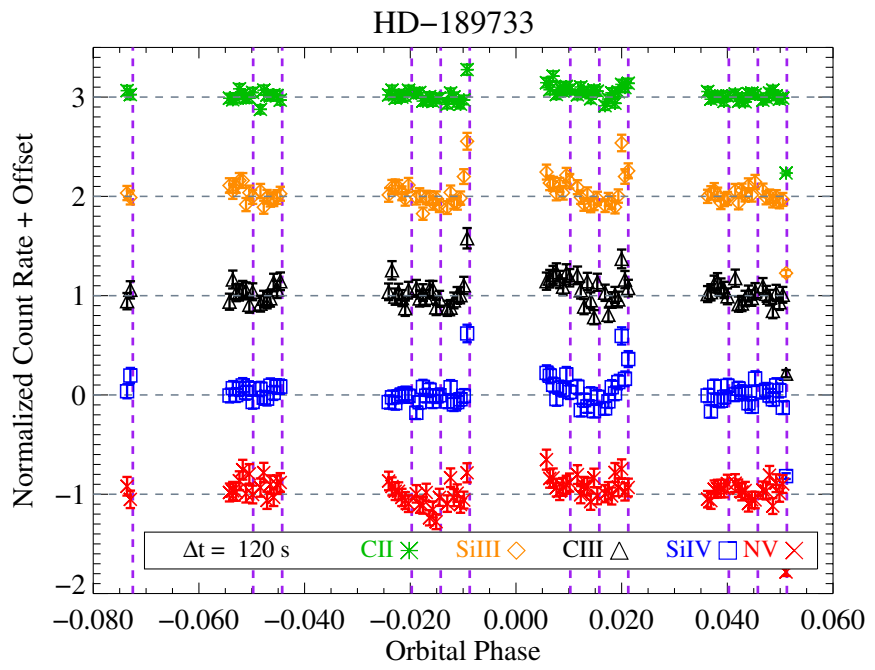

Figure 23. Count rates of the brightest far-UV emission lines not contaminated by geocoronal airglow (e.g., $\mathrm{H}_{\mathrm{I}} \mathrm{Ly} \alpha$ and $\left.\mathrm{O}_{\mathrm{I}} \lambda 1304\right)$. The light curves were created from the two-dimensional COS data files, with the total number of counts in given line (defined by an $[x, y]$ location on the COS detector) integrated in $120 \mathrm{~s}$ intervals. Error bars are taken to be the square root of the number of counts in a given time bin. We note that the variability on minute to hour timescales exceeds the photon noise and can be the limiting factor for transit studies of chromospherically active, low-mass stars. The dashed lines denote the end of an individual COS exposure.

(A color version of this figure is available in the online journal.)

As COS is a slitless spectrograph, geocoronal emission complicates observations at discrete wavelengths (most notably H I Ly $\alpha, 1216 \AA$, and the O I multiplet at $1304 \AA$ ). However, the high sensitivity and low instrumental background allow high-S/N observations in emission lines of several other chromospheric and transition region ions, including C III $\lambda 1175$, Si III $\lambda 1206, \mathrm{~N} v \lambda 1240, \mathrm{C}$ II $\lambda 1335$, and Si IV $\lambda 1400$. Unfortunately, the very processes powering these emission lines (e.g., Ayres \& France 2010) also limit the capability of far-UV transit studies.

Light curves of the bright chromospheric emission lines from the spectrum in Figure 22 were created from the calibrated two-dimensional data by exploiting the TIME-TAG capability (France et al. 2010). These are shown in Figure 23. We extracted an $\left[x_{i}, y_{i}, t_{i}\right]$ photon list from each exposure $i$ and co-added these to create a master $[x, y, t]$ photon list. The total number of counts in a $[\Delta x, \Delta y]$ box was integrated over a timestep of $120 \mathrm{~s}$. The instrument background level is computed in a similar manner, with the background integrated over the same wavelength interval as the emission lines, but offset below the active science region. The instrumental background is $<6 \%$ of the total line flux for all species presented in Figure 23. Following the extraction of the light curves in photons $\mathrm{s}^{-1}$, the data are normalized for a relative comparison of the line flux as a function of time. For each species, we created a linear fit to the OOT light curves (orbital phase $<-0.015$ and $>0.030$ ), excluding time bins at the beginning or end of an orbit. This is similar to the normalization procedure adopted in Paper I and in Figure 2. These normalized light curves are plotted in Figure 23. The variation on timescales of minutes to hours is significantly larger than the statistical error in an individual bin.

If we assume that exposures taken on the fourth spacecraft orbit represent the in-transit spectrum, then we can compare the co-added, one-dimensional in-transit and OOT spectra. Integrating the total flux over airglow-free wavelength intervals, $\Delta \lambda=[1150-1210 \AA, 1220-1270 \AA, 1308-1425 \AA]$, we find 
an in-transit/OOT flux ratio of $1.000 \pm 0.017$. These FUV observations therefore give no detection of the transit. Other FUV transit observations have given more reliable information, partly because HD 209458 is an older, solar-type star with less activity than HD 189733, but any FUV light curve results from the unknown and unreproducible locus of the transit light curve across the patchy and changing stellar chromospheric emission distribution (Haswell 2010). Lecavelier Des Etangs et al. (2012) report STIS FUV observations of two transits of HD 189733 b. They too fail to detect the transit in observations made in 2010 April. In 2011 September, the STIS observations were accompanied by contemporaneous X-ray observations in which stellar flaring activity was detected. This time the Ly $\alpha$ profile showed variations attributed to exospheric absorption, but no signal was detected in Si III, O v, or N v. Lecavelier Des Etangs et al. (2012), perhaps because of the brevity required for a Letter, give no assessment of the variability inherent in the observed stellar Ly $\alpha$ emission. Intriguingly, the X-ray light curve of Lecavelier Des Etangs et al. (2012) shows a high point during optical ingress that is reminiscent of our Visit 2 NUVA and NUVC orbit 3 anomaly.

Like the FUV observations of HD 189733, our Visit 2 NUV light curve of WASP-12 was also complicated by stellar activity, but we were able to isolate and ameliorate its effect (cf. the NUVA light curve in Figure 9). The NUV can provide more robust information on absorption by diffuse gas than the FUV. The COS/FUV observations of HD 209458 b (Linsky et al. 2010) have exquisitely high S/N; NUV observations of this planet would clearly reveal much information about the composition and velocity structure of its exosphere.

\subsection{Summary}

1. We observed WASP-12 in the NUV, a spectral region which proves to be an extremely sensitive probe for the presence of absorbing gas.

2. We have detected asymmetric absorbing material in the WASP-12 system, which is more extensive than the Roche lobe of WASP-12 b.

3. The stellar photospheric absorption appears to be a fairly good predictor of the wavelength dependence of the excess absorption, indicating that the extended gas may be similar in composition and ionization state to the WASP-12 photosphere. The NUVB spectral region which has the least photospheric absorption and is devoid of strong resonance lines yields the most straightforward light curve. This gives us confidence in our interpretation.

4. In HST Visit 1, we detected enhanced absorption during and before the optical transit.

5. In HST Visit 2, our NUV light curve appeared to be below the OOT flux level for all the pre-ingress coverage, despite this extending to earlier phases than that of Visit 1. Thus, the spatial distribution of the absorbing gas appears to vary.

6. The cores of the $\mathrm{Mg}$ II and Fe II resonance lines are very dark throughout our coverage. WASP-12 would be completely unique among all stars of similar age and spectral type if this lack of observed $\mathrm{Mg}$ II chromospheric emission reversals was intrinsic to the star. We attribute it instead to absorption occurring in gas between us and WASP-12. The required column density is at least $\sim 2 \times 10^{17} \mathrm{~cm}^{-2}$. This value is about a factor of 10 greater than a plausible estimate of the interstellar column density, but is consistent with gas lost from WASP-12 b via photo-evaporation. Nevertheless, the ISM explanation cannot be dismissed completely, since there is substantial "cosmic variance" in ISM properties, and the WASP-12 sight line might just be unusually opaque in $\mathrm{Mg}$ II.

7. The lack of Mg II chromospheric emission cores from the star could be explained by absorbing gas not confined to the region immediately surrounding the planet. Absorbing gas would need to occupy the lines of sight to at least the midlatitude regions of the stellar disk at all observed orbital phases. We show that an outflow of some fraction of the mass loss expected from WASP-12 b can plausibly provide this obscuration, though we have not proved that the gas will necessarily fulfill the geometric requirements.

8. In $H S T$ Visit 2, the NUV flux was near its maximum value at optical ingress. Such a fast change in flux is a signature of a stellar flare, although such an event would be very rare on a low-activity star like WASP-12. Nevertheless, there are (albeit very noisy) hints in the $\mathrm{Mg}$ II line profile of some chromospheric emission at this time.

9. FUV transit observations can be far more affected by stellar activity than our NUV observations were. In the NUV, we have the opportunity to isolate the effects of activity by excluding spectral lines that are strongly affected.

10. We obtained new optical photometry and combined this with archive optical photometry to derive an improved linear ephemeris:

$$
\begin{aligned}
T_{\text {mid }}(\text { HJD })= & 2454852.7739_{-0.00014}^{+0.00014} \\
& +N \times 1.09142206_{-0.000000031}^{+0.0000003}
\end{aligned}
$$

\subsection{Concluding Remarks}

Given its spectral type and probable age, we have suggested that WASP-12 should have a normal level of chromospheric activity, despite the lack of observed emission cores in the Mg II resonance lines. This hypothesis, in principle, could be tested by obtaining far-UV (FUV) spectra, which should show the normal complement of chromospheric (e.g., O I $\lambda 1305$ triplet; C II $\lambda 1335$ multiplet) and higher-temperature features (e.g., C IV $\lambda 1550$ doublet). However, the great distance of WASP12 (at least 300 pc; Fossati et al. 2010b) would imply line peak flux densities below $10^{-16} \mathrm{erg} \mathrm{cm}^{-2} \mathrm{~s}^{-1} \AA^{-1}$ (scaling from the FUV spectrum of $\alpha$ Cen A), which even with the high sensitivity G140L grating of COS would require an exposure of $\sim 25 \mathrm{ks}$ to reach a secure $\mathrm{S} / \mathrm{N} \sim 10$, or nearly 10 HST orbits. Even worse, the estimated interstellar reddening of $E(B-V) \sim 0.2$ mag would imply an additional factor of $\sim 5$ attenuation of the FUV lines, further lengthening the necessary COS or STIS exposures to beyond the realm of practicality. Furthermore, this does not even account for the additional absorption deduced from the WASP-12 Mg II lines, which - if truly interstellar and accompanied by the normal dustto-gas ratio-would completely extinguish the FUV radiation. Similarly, and again scaling from $\alpha$ Cen A, WASP-12 would be predicted to be a 0.02 counts $\mathrm{ks}^{-1}$ coronal X-ray source in the Chandra imagers, which is about a factor of two below the average cosmic background rate. In that background-limited regime, it would require at least $1 \mathrm{Ms}$ of exposure to achieve a minimal $3 \sigma$ detection, again completely impractical.

We need to look at more close-in exoplanets using this highly informative NUV wavelength region. Most known hot 
Jupiters are less distant than WASP-12 and consequently will offer higher NUV fluxes and thus better signal to noise. Our conclusions throughout this paper are limited by the noise level in our data.

Many related studies have been performed in the FUV. The FUV does provide access to higher ionization state lines that can be good proxies for activity and suffer less confusion with the lower-ionization ISM (C III, N v, Si IV). Additionally, the C III FUV multiplet is not a resonant transition, its lower level lies about $6.5 \mathrm{eV}$ above the ground state, therefore this line is effectively never seen in the diffuse ISM. Despite this, as Figure 5.10 of Haswell (2010) makes clear, the NUV has some clear advantages over the FUV for studies of exospheric absorbing gas. NUV transits are measured against the relatively uniform and well-understood stellar photospheric light, whereas the FUV emission from the stellar disk is patchy, time variable, and limited to the wavelengths of strong chromospheric emission lines. In addition to the consequent improvement in robustness of the inferences drawn about the absorbing gas, the NUV also has a huge advantage in stellar flux and thus $\mathrm{S} / \mathrm{N}$. There are many open questions and much theoretical and modeling activity connected with the handful of extant UV observations of transiting hot Jupiters. It is vital that more UV transit observations are made while $H S T$ remains available.

We gratefully acknowledge discussions with Moira Jardine, Christiane Helling, and Aline Vidotto. This work is based on observations made with the NASA/ESA Hubble Space Telescope, obtained from MAST at the Space Telescope Science Institute, which is operated by the Association of Universities for Research in Astronomy, Inc., under NASA contract NAS 526555. These observations are associated with program 11651 to which support was provided by NASA through a grant from the Space Telescope Science Institute. This work was supported by an STFC Rolling Grant (C.A.H., L.F., U.C.K.). R.B. is supported by an STFC studentship.

Facilities: HST (COS), FTN

\section{REFERENCES}

Adams, F. C. 2011, ApJ, 730, 27

Ayres, T. 2010, Mem. Soc. Astron. Ital., 81, 553

Ayres, T., \& France, K. 2010, ApJ, 723, L38

Ben-Jaffel, L. 2007, ApJ, 671, L61

Chan, T., Ingemyr, M., Winn, J. N., et al. 2011, AJ, 141, 179

Collier Cameron, A., Wilson, D. M., West, R. G., et al. 2007, MNRAS, 380,1230

Danforth, C. W., Keeney, B. A., Stocke, J. T., Shull, J. M., \& Yao, Y. 2010, ApJ, 720,976

Desert, J.-M., Vidal-Madjar, A., Lecavalier des Etangs, A., et al. 2008, A\&A, 492,585

Ehrenreich, D., \& Desert, J.-M. 2011, A\&A, 529, A136
Ekenbäck, A., Holmström, M., Wurz, P., et al. 2010, ApJ, 709, 670

Enoch, B., Collier Cameron, A., Parley, N. R., \& Hebb, L. 2010, A\&A, 516, 33

Erkaev, N. V., Kulikov, Yu. N., Lammer, H., et al. 2007, A\&A, 472, 329

Fortney, J. J., Shabram, M., Showman, A. P., et al. 2010, ApJ, 709, 149

Fossati, L., Bagnulo, S., Elmasli, A., et al. 2010a, ApJ, 720, 872

Fossati, L., Haswell, C. A., Froning, C. S., et al. 2010b, ApJ, 714, L222

France, K., Linsky, J. L., Brown, A., Froning, C. S., \& Béland, S. 2010, ApJ, 715,596

Green, J. C., Froning, C. S., Osterman, S., et al. 2012, ApJ, 744, 60

Guo, J. H. 2011, ApJ, 733, 98

Haswell, C. 2010, Transiting Exoplanets (Cambridge: Cambridge University Press), CUP ISBN 978-0521139380

Hebb, L., Collier-Cameron, A., Loeillet, B., et al. 2009, ApJ, 693, 1920

Holmes, S., Kolb, U., Haswell, C. A., et al. 2011, PASP, 123, 1177

Holmström, M., Ekenbäck, A., Selsis, F., et al. 2008, Nature, 451, 970

Husnoo, N., Pont, F., Hébrard, G., et al. 2011, MNRAS, 413, 2500

Irwin, M., \& Lewis, J. 2001, NewAR, 45, 105

Knutson, H. A., Charbonneau, D., Allen, L. E., et al. 2007, Nature, 447, 183

Knutson, H. A., Howard, A. W., \& Isaacson, H. 2010, ApJ, 720, 1569

Kupka, F., Piskunov, N., Ryabchikova, T. A., Stempels, H. C., \& Weiss, W. W. 1999, A\&AS, 138, 119

Lai, D., Helling, Ch., \& van den Heuvel, E. P. J. 2010, ApJ, 721, 923

Lecavelier Des Etangs, A. 2007, A\&A, 461, 1185

Lecavelier Des Etangs, A., Bourrier, V., Wheatley, P. J., et al. 2012, A\&A, 543, L4

Lecavelier Des Etangs, A., Ehrenreich, D., Vidal-Madjar, A., et al. 2010, A\&A, 514,72

Lecavelier Des Etangs, A., Vidal-Madjar, A., McConnell, J. C., \& Hebrard, G. 2004, A\&A, 418,

Li, S., Miller, N., Lin, D. N. C., \& Fortney, J. J. 2010, Nature, 463, 1054

Linsky, J. L., \& Wood, B. E. 1996, ApJ, 463, 254

Linsky, J. L., Yang, H., France, K., et al. 2010, ApJ, 717, 1291

Llama, J., Wood, K., Jardine, M., et al. 2011, MNRAS, 416, L41

Lopez-Morales, M., Coughlin, J. L., Sing, D. K., et al. 2010, ApJ, 716, L36

Maciejewski, G., Errmann, R., Raetz, St., et al. 2011, A\&A, 528, A65

Moses, J. I., Visscher, C., Fortney, J. J., et al. 2011, ApJ, 737, 15

Osten, R., Ghavmian, P., Nemi, S.-M., et al. 2010, Instrument Science Report COS 2010-15 (v1) (Baltimore, MD: STScI)

Osten, R., Massa, D., Bostroem, A., Aloisis, A., \& Proffitt, C. 2011, Instrument Science Report COS 2011-02 (Baltimore, MD: STScI)

Osterman, S., Green, J., Froning, C., et al. 2011, Ap\&SS, 335, 257

Piskunov, N. E., Kupka, F., Ryabchikova, T. A., Weiss, W. W., \& Jeffery, C. S 1995, A\&AS, 112, 525

Raiput, J., \& Safvan, C. P. 2011, J. Chem. Phys., 134, 201101

Ryabchikova, T. A., Piskunov, N. E., Stempels, H. C., Kupka, F., \& Weiss, W. W. 1999, Phys. Scr., T83, 162

Savage, B. D., \& Mathis, J. S. 1979, ARA\&A, 17, 73

Schroder, C., Reiners, A., \& Schmitt, J. H. M. M. 2009, A\&A, 493, 1099

Shulyak, D., Tsymbal, V., Ryabchikova, T., Stütz, Ch., \& Weiss, W. W. 2004, A\&A, 428, 993

Spiegel, D. S., Burrows, A., Ibgui, L., Hubeny, I., \& Milsom, J. A. 2010, ApJ, 709,149

Tremblin, P., \& Chiang, E. 2012, arXiv:1206.5003

Vidal-Madjar, A., Désert, J.-M., Lecavelier des Etangs, A., et al. 2004, ApJ, 604, L69

Vidal-Madjar, A., Lecavelier des Etangs, A., Désert, J.-M., et al. 2003, Nature, 422,143

Vidal-Madjar, A., Lecavelier des Etangs, A., Désert, J.-M., et al. 2008, ApJ, 676, L57

Vidotto, A. A., Jardine, M., \& Helling, Ch. 2010, ApJ, 722, 168

Vidotto, A. A., Jardine, M., \& Helling, Ch. 2011, MNRAS, 414, 1573 